Research paper

\title{
A continuous contact force model for impact analysis in multibody dynamics
}

\author{
Jie Zhang ${ }^{\mathrm{a}}$, Wenhao $\mathrm{Li}^{\mathrm{b}}$,c , Lei Zhao ${ }^{\mathrm{a}}$, Guangping $\mathrm{He}^{\mathrm{a}, *}$ \\ a School of Mechanical and Materials Engineering, North China University of Technology, No.9, Jinyuanzhuang Road, Shijingshan District, \\ Beijing 100144, China \\ b Institute of Mechanics, Chinese Academy of Sciences, No.15 Beisihuanxi Road, Haidian District, Beijing 100190, China \\ ' School of Engineering Science, University of Chinese Academy of Sciences, No.19(A) Yuquan Road, Shijingshan District, Beijing 100049, \\ China
}

\section{A R T I C L E I N F O}

\section{Article history:}

Received 12 April 2020

Revised 2 May 2020

Accepted 3 May 2020

Available online 12 June 2020

\section{Keywords:}

Contact force model

Energy loss

Complex geometries

Multibody dynamics

\begin{abstract}
A B S T R A C T
A new continuous contact force model for contact problems with complex geometries and energy dissipations in multibody systems is presented and discussed in this work. The model is developed according to the Hertz law, and a hysteresis damping force is introduced for modeling the energy dissipation during the contact process. As it is almost impossible to obtain an analytical solution based on the system dynamic equation, an approximate dynamic equation for the collision system is proposed, achieving a good approximation of the system dynamic equation. An approximate function relating the deformation velocity and indentation depth is determined based on the approximate dynamic equation and is utilized to calculate the energy loss due to the damping force. Then, a primary formula of the hysteresis damping parameter is derived by combining energy balance and the law of conservation of linear momentum. The new model is developed by modifying the primary formula through nondimensional analysis. The comparison with published experimental data and the analysis of the simulation data of eight different continuous contact models reveal the capability and high precision of the new model as well as the effect of the geometry of the contacting surfaces on the dynamic system response.
\end{abstract}

(c) 2020 Elsevier Ltd. All rights reserved.

\section{Introduction}

Contact-impact phenomena frequently occur in multibody systems, mainly due to the clearances between bodies and joints [1-7]. Proper modeling of a contact-impact phenomenon is very important for an accurate description of the dynamic behavior of multibody systems [8,9]. In the past few years, research on impact analysis in multibody systems has increased significantly $[1,9,10]$. The contact-impact process is characterized by an extremely short duration, a large contact force, fast energy dissipation, and great changes in the body velocities [11]. The modeling of contact-impact problems relies heavily on several factors, such as the topological properties of contacting surfaces, material characteristics, initial velocities and friction [12]. To describe the dynamic behavior of a multibody system, several crucial aspects of the impact modeling need to be taken into account, including the change in velocity before and after the impact, the duration time, the peak contact force and the indentation depth [9].

\footnotetext{
* Corresponding author.

E-mail address: hegp55@126.com (G. He).
} 


\section{Nomenclature}

\begin{tabular}{|c|c|}
\hline $\begin{array}{l}i, j \\
v_{i}^{(-)}, v_{j}^{(-)}\end{array}$ & $\begin{array}{l}\text { impactor, target } \\
\text { initial velocity }\end{array}$ \\
\hline$v_{i}^{(+)}, v_{j}^{(+)}$ & separation velocity \\
\hline $\begin{array}{l}\mathrm{t}^{(-)}, \mathrm{t}^{(+)}, t_{m} \\
\delta, \delta_{m} \\
v_{i j}\end{array}$ & $\begin{array}{l}\text { time of initial contact, time of separation, time of maximum indentation } \\
\text { deformation or indentation, maximum indentation } \\
\text { velocity of the objects at time } t_{\mathrm{m}}\end{array}$ \\
\hline$\dot{\delta}, \ddot{\delta}$ & deformation velocity or indentation velocity, indentation acceleration \\
\hline$m, k$ & equivalent mass, equivalent stiffness \\
\hline$F_{N}, F_{\max }$ & normal contact force, maximum of the normal contact force \\
\hline$n$ & non-linear power exponent \\
\hline$c_{r}$ & coefficient of restitution \\
\hline$\lambda$ & hysteresis damping factor \\
\hline$\dot{\delta}^{(-)}, \dot{\delta}^{(+)}$ & initial indentation velocity, relative separation velocity \\
\hline$\dot{\delta}, \dot{\delta}_{c}, \dot{\delta}_{r}$ & equivalent velocity, equivalent velocity of compression phase, equivalent velocity of restitution phase \\
\hline $\begin{array}{l}T, t_{c}, t_{r} \\
\Delta E_{\text {loss }}\end{array}$ & $\begin{array}{l}\text { time of the whole contact phase, compression phase and restitution phase } \\
\text { energy loss }\end{array}$ \\
\hline$\Delta \mathrm{E}_{c}, \Delta \mathrm{E}_{r}$ & energy loss for compression or restitution phase calculating based on the system dynamic equation \\
\hline$\Delta E_{c}^{*}, \Delta E_{r}^{*}$ & energy loss for compression or restitution phase calculating based on the approximate dynamic equation \\
\hline$x, \tau$ & non-dimensional variables \\
\hline$\alpha$ & modification parameter \\
\hline$k^{*}, \lambda^{*}$ & non-dimensional parameters \\
\hline$\beta$ & non-linear power exponent associated with damping \\
\hline
\end{tabular}

The earliest model of impact is the coefficient of restitution, which can describe the changes in the velocity and energy before and after the impact. There are different definitions of the coefficient of restitution $[13,14]$. Based on the speed before and after the impact, Newton's definition is the most popular and commonly used among them [10]. The restitution coefficient, which is relatively easy to measure experimentally [15], provides a concise description of the impact phenomenon. However, the details of the contact force and deformation in the process of collision cannot be described by the coefficient of restitution, except for the velocity change and the energy loss before and after the impact.

The second approach is the nonsmooth method, in which the duration of the collision is ignored, and the impact is assumed to occur instantaneously [16]. There are two ways to treat contact-impact problems in a multibody system, namely, the linear complementarity problem (LCP) $[17,18]$ and differential variational inequality (DVI) $[19,20]$. Compared with the coefficient of restitution, the nonsmooth approach can calculate the contact force with a relatively efficient calculation; however, this approach is not valid for the modeling of impact duration due to the instantaneous assumption [9,21-23].

The third approach is named the compliant continuous contact force model, because the contact force is described as a continuous function of the indentation depth (relative deformation). Time histories of velocities contact forces and deformations, and duration time can be described by this method. The nonlinear Hertz contact model proposed in 1880 has provided an important basis for fundamental research on contact mechanics [24]. In recent years, several continuous contact models considering the energy dissipation and impact duration have been proposed on the basis of the Hertz model [25,26], such as the influential models proposed by Hunt and Crossley [27], Lankarani and Nikravesh [21], and Flores et al. [11]. Other continuous contact models, such as the Herbert and McWhannell model [28], the Lee and Wang model [29], the Gonthier et al. model [30], the Zhiying and Qishao model [31], the Kuwabara and Kono model [32], the Gharib and Hurmuzlu model [33], the Hu and Guo model [12], the Shen et al. model [34], the Safaeifar and Farshidianfar model [35], the Yu et al. model [36], the Poursina and Nikravesh model [37], the Carvalho and Martins model [38] and the Jian et al. model [39], have been developed. In these models, the hysteresis damping factor is derived as a function of the restitution coefficient, which is relatively easy to measure [15]. The strengths and weaknesses of these models have been discussed in the literature [1,9], especially the application limitations [40].

A new continuous contact force model, which is inspired by the work of Flores et al. [10] and Hu and Guo [12], is proposed in this paper. As it is almost impossible to obtain an analytical solution based on the system dynamic equation, an approximate dynamic equation for the collision system is proposed for the first time, achieving a good approximation of the system dynamic equation. The new model is established via nondimensional analysis and is likely to solve the contact problems concerning complex geometries. The comparison with experimental data and the analysis of simulation data show a very high accuracy of the new model, which will be useful for multibody system dynamics. The remainder of the paper is organized as follows. Section 2 covers the fundamentals of the continuous contact force models. Then, the energy loss associated with the restitution coefficient is described in Section 3. Section 4 demonstrates the construction of the new contact model, and the model is verified in Section 5 through the comparison with experimental data and the analysis of 


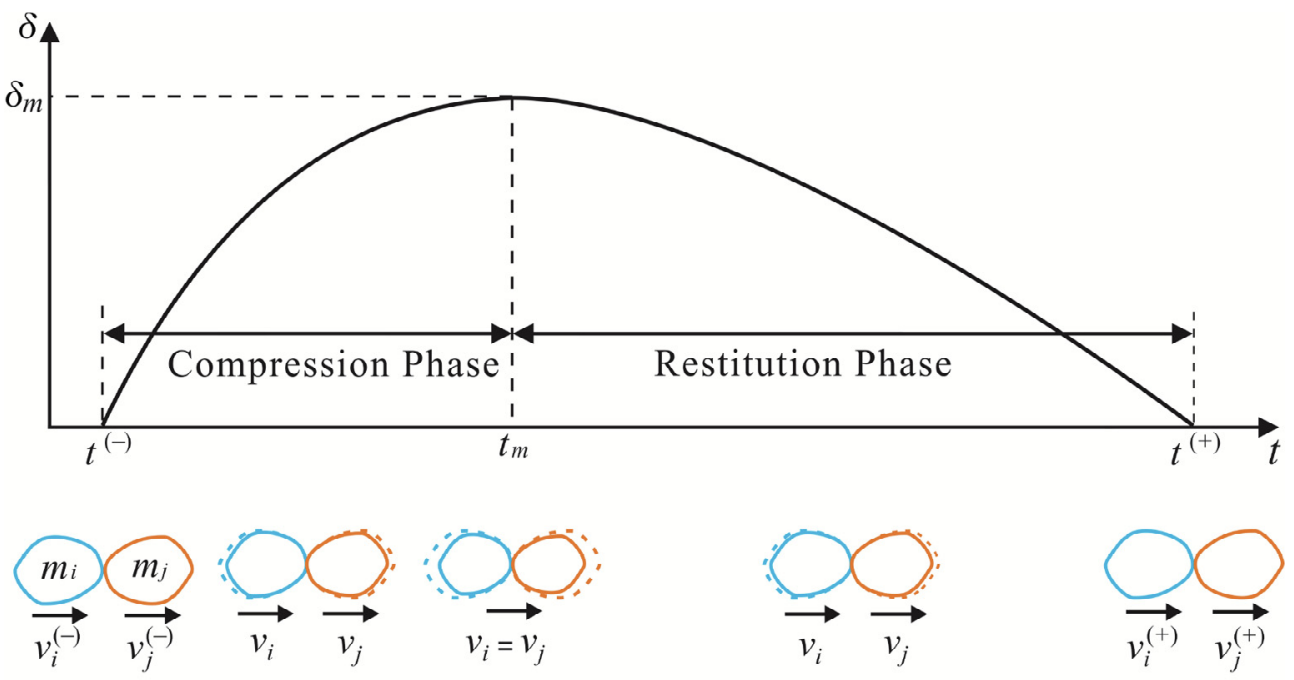

(a)
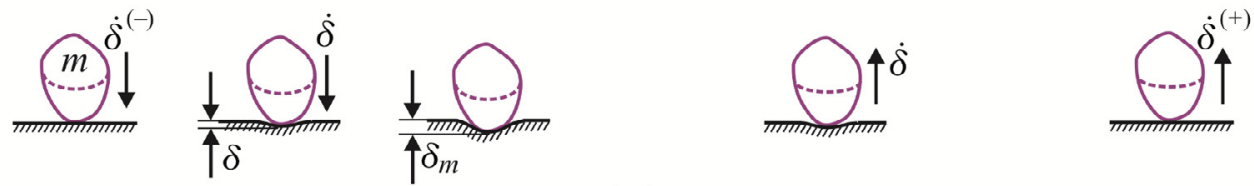

(b)

Fig. 1. Contact process between two impacting objects and the equivalent system.

simulation data of eight different contact models. Numerical examples and conclusions are presented in Sections 6 and 7 , respectively.

\section{General issues regarding the continuous contact force models}

As shown in Fig. 1a, two solid objects (with masses $m_{i}$ and $m_{j}$ ) with a direct-central impact, the deformation taking place in the local contact zone, is divided into two phases: the compression phase and the restitution phase. At the initial time of impact $\mathrm{t}^{(-)}$, the objects have velocities $v_{i}^{(-)}$and $v_{j}^{(-)}$; then, the deformation increases until the relative normal deformation between the contacting bodies reaches the maximum $\delta_{m}$ at time $t_{m}$, and the objects reach the same velocity $v_{i j}$. After that, the deformation begins to recover, the contact force gradually decreases until it becomes zero at time $\mathrm{t}^{(+)}$, and the velocities become $v_{i}^{(+)}$and $v_{j}^{(+)}$. The period from $\mathrm{t}^{(-)}$to $\mathrm{t}_{\mathrm{m}}$ is the compression phase, and the restitution phase starts from tm and ends at $\mathrm{t}^{(+)}$, as shown in Fig. 1a. When the size of the contact area is much smaller than the sizes of the contacting objects, the contact-impact system with two objects can be equivalent to a collision between an object with equivalent mass $m$ and an elastic half space [7,27], as illustrated in Fig. 1b. This equivalence is valid for most elastic contact-impact problems $[41,42]$.

The groundbreaking work of Hertz on contact problems has continued to be an important basis for both fundamental research and engineering applications of contact mechanics. Based on elastic mechanics, the Hertz model described the relationship between the normal contact force $F_{N}$ and the indentation depth $\delta$, as shown in Eq. (1) [27],

$$
F_{N}=k \delta^{n}
$$

where $k$ represents the generalized stiffness parameter and $\delta$ is the indentation caused by deformation, as illustrated in Fig. 1b. The exponent $n$ depends on the topological properties of the contacting surfaces [43]. Theoretical analysis shows that for the contact between a sphere, cube, prism, cylinder (horizontal and vertical), or cone and an elastic half space, the $n$ value is $1.5,1.0,1.0,1.0,1.0$ or 2.0, respectively [42], as shown in Fig. 2. The spherical contact is the most representative among them [10], so in most studies $\mathrm{n}$ is considered to be 1.5. Of course, this assumption is not applicable in all situations; for instance, in some cases, $\mathrm{n}$ is considered approximately equal to 2.0 for grains with irregular surfaces such as sand [44]. In general, the exponent $n$ is variable and depends on the contact surface geometry.

Although the energy dissipation during collision is neglected by the Hertz model [45], which is based on elasticity theory, the literature [46] has pointed out that even though $40 \%$ of the kinetic energy is lost due to viscous dissipation during the collision, Hertz's theory is still accurate in predicting the collision time and the maximum contact area. Therefore, the 


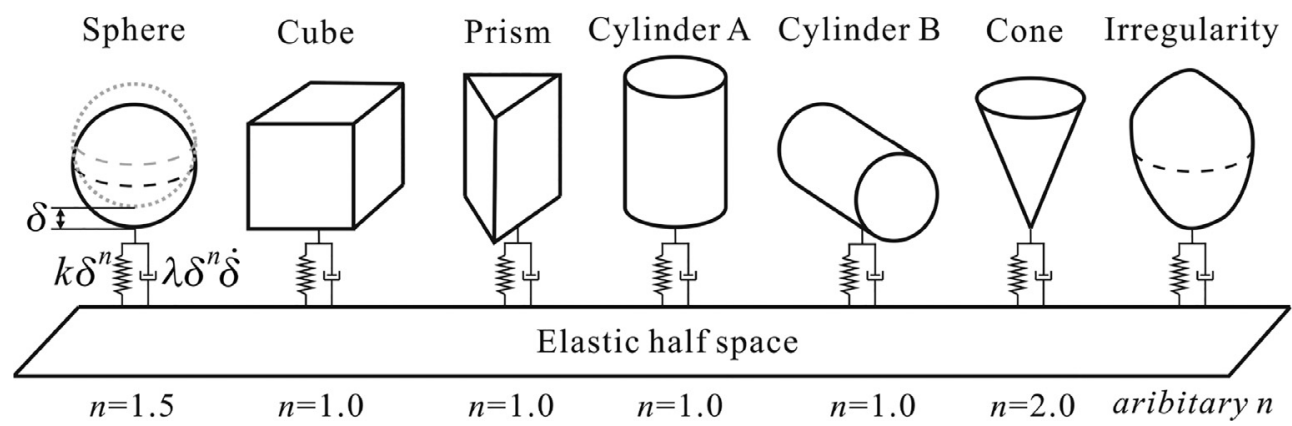

Fig. 2. Equivalent contact problems with multiple contacting surfaces.

Hertz model can be used as a basis for contact problems with energy dissipation, and several continuous contact models considering energy dissipation during collision have been proposed. In these models, all the energy losses that occur during the collision are assumed to be taken by the viscous damper [10,47], and the contact system is equivalent to a singledegree-of-freedom dynamic system with a nonlinear spring and nonlinear damping, as shown in Fig. 2 . The contact force is composed of an elastic force and a dissipative force, and the exponent of the damping coefficient is generally considered to be equal to the exponent of the indentation depth proposed by Hunt and Crossley [27]; consequently, the expression of the contact force is

$$
F_{N}=k \delta^{n}+\lambda \delta^{n} \dot{\delta}
$$

where $\dot{\delta}$ is the deformation velocity, $\lambda$ is the hysteresis damping factor. Theoretical analysis shows that $\lambda$ is a function of the equivalent stiffness $k$, the coefficient of restitution, and the initial relative velocity. Compared to the Kelvin-Voigt model with linear damping, the models with nonlinear damping are more accurate and avoid a nonzero contact force at zero deformation. Therefore, the expression form of the contact force described in Eq. (2) is considered the basis of most continuous contact force models $[1,9,26]$, including the model proposed in our study.

\section{The energy loss associated with the restitution coefficient}

For a more accurate model, the energy change during the collision process needs to be considered [48]. The description of the energy change utilizes the coefficient of restitution, which is relatively easy to measure [15] but cannot describe the contact force, deformation, etc. of the collision process.

The coefficient of restitution, denoted $c_{r}$, is defined $[10,12]$ as

$$
c_{r}=-\frac{\dot{\delta}^{(+)}}{\dot{\delta}(-)}
$$

where $\dot{\delta}^{(-)}=v_{i}^{(-)}-v_{j}^{(-)}$and $\dot{\delta}^{(+)}=v_{i}^{(+)}-v_{j}^{(+)}$are the initial relative velocity and the relative separating velocity respectively.

According to energy balance and the law of conservation of linear momentum in the period from $\mathrm{t}^{(-)}$to $\mathrm{t}^{(+)}$, the following expressions are acquired

$$
\begin{aligned}
& {\left[\frac{1}{2} m_{i}\left(v_{i}^{(-)}\right)^{2}+\frac{1}{2} m_{j}\left(v_{j}^{(-)}\right)^{2}\right]=\Delta E_{\text {loss }}+\left[\frac{1}{2} m_{i}\left(v_{i}^{(+)}\right)^{2}+\frac{1}{2} m_{j}\left(v_{j}^{(+)}\right)^{2}\right]} \\
& m_{i} v_{i}^{(-)}+m_{j} v_{j}^{(-)}=m_{i} v_{i}^{(+)}+m_{j} v_{j}^{(+)}
\end{aligned}
$$

The expression of the total amount of energy loss $\Delta E_{\text {loss }}$ can be obtained through Eqs. (4) and (5):

$$
\Delta E_{\text {loss }}=\frac{1}{2} m\left[\left(v_{i}^{(-)}-v_{j}^{(-)}\right)^{2}+\left(v_{i}^{(+)}-v_{j}^{(+)}\right)^{2}\right]
$$

where $m$ is the equivalent mass of the contact system, which is defined as $m=\frac{m_{i} m_{j}}{m_{i}+m_{j}}$.

Combined with the definition of the restitution coefficient $c_{r}$ described in Eq. (3), Eq. (6) can be simplified as

$$
\Delta E_{\text {loss }}=\frac{1}{2} m\left(1-c_{r}^{2}\right) \dot{\delta}^{(-) 2}
$$




\section{The development of the new model}

\subsection{General issues regarding the construction of the new model}

Although the coefficient of restitution can describe the changes in energy and velocity before and after the collision concisely, some important details of the contact-impact process cannot be obtained, as mentioned above. Previous studies have shown that continuous contact models can depict these details if the hysteresis damping factor $\lambda$ is fixed by the measured value of the restitution coefficient. As one continuous contact model was adopted to simulate the contact-impact process, the simulation value of the restitution coefficient can be calculated by the simulation values of the velocities before and after the collision. In this research, the set value of the restitution coefficient, which is provided as input to the contact force models for calculating the hysteresis damping factor, is defined as the pre-restitution coefficient, and the simulation value of the restitution coefficient is defined as the post-restitution coefficient. From a physical point of view, the smaller the difference between the post- and pre-restitution coefficients, the more closely the collision process described by the model matches the actual situation [12], resulting in a more accurate description of the dynamic behavior of multibody systems.

According to the expression of impact force described in Eq. (2), the dynamic equation for the contact-impact system as illustrated in Figs. 1 and 2 is represented below [10,12,21,27,34]:

$$
m \ddot{\delta}+k \delta^{n}+\lambda \delta^{n} \dot{\delta}=0
$$

where gravity is ignored because it is much smaller than the impact force.

The determination of the expression of $\lambda$ is the key step of the construction of the contact model. It is almost impossible to obtain an analytical solution to Eq. (8), and several methods have been applied to derive the approximate expression of the damping coefficient $\lambda$ in previous studies [1,10,12,21,27-31]. In recent continuous contact model studies, new contact force models were provided [10,12], considering the approximate functions between the deformation velocity and indentation depth for the case of $n=1.5$. Both models produce results that represent the actual situation better than other models do, in terms of the difference between the post- and pre-restitution coefficients [1,12], especially for soft materials with low restitution coefficients [10].

Inspired by the above research on constructing a contact force model, this article attempts to provide a new continuous contact model on the foundation of an approximate dynamic equation for contact problems with more complex geometries, which means that the exponent $n$ is arbitrary.

\subsection{Approximate dynamic equation for the impact system}

The system dynamic equation as expressed in Eq. (8) can be rewritten as

$$
m \ddot{\delta}+(k+\lambda \dot{\delta}) \delta^{n}=0
$$

Let us consider an equation similar to Eq. (9):

$$
m \ddot{\delta}+(k+\lambda \hat{\dot{\delta}}) \delta^{n}=0
$$

where $\widehat{\dot{\delta}}$ is the equivalent velocity, which remains constant during the compression and restitution phases and is associated with $\dot{\delta}^{(-)}$. The details will be discussed in the following section.

By integrating Eq. (10) over the compression phase, it can be derived that,

$$
\dot{\delta}^{2}-\dot{\delta}^{(-) 2}=-\frac{2(k+\lambda \widehat{\dot{\delta}})}{m(n+1)} \delta^{n+1}
$$

If the deformation velocity $\dot{\delta}=0$, the deformation $\delta$ will be reached the maximum value $\delta_{m}$, substituting $\dot{\delta}=0$ into Eq. (11), it can be derived that,

$$
\delta_{m}^{n+1}=\frac{m(n+1)}{2\left(k+\lambda \widehat{\dot{\delta}}_{c}\right)} \dot{\delta}^{(-) 2}
$$

The function between deformation velocity $\dot{\delta}$ and indentation depth $\delta$ during compression phase can be acquired from Eqs. (11) and (12),

$$
\dot{\delta}=\dot{\delta}^{(-)} \sqrt{1-\left(\frac{\delta}{\delta_{m}}\right)^{n+1}}
$$


Then, this equation is applied to calculate the work done by the dissipative force during the compression phase according to Eq. (2):

$$
\begin{aligned}
\Delta \mathrm{E}_{c} & =\int_{0}^{\delta_{m}} \lambda \delta^{n} \dot{\delta} d \delta=\int_{0}^{\delta_{m}} \lambda \delta^{n} \dot{\delta}^{(-)} \sqrt{1-\left(\frac{\delta}{\delta_{m}}\right)^{n+1}} d \delta \\
& =\int_{0}^{\delta_{m}} \frac{\lambda \delta_{m}^{n+1} \dot{\delta}^{(-)}}{n+1} \sqrt{1-\left(\frac{\delta}{\delta_{m}}\right)^{n+1}} d\left(\frac{\delta}{\delta_{m}}\right)^{n+1} \\
& =\int_{0}^{1} \frac{\lambda \delta_{m}^{n+1} \dot{\delta}^{(-)}}{n+1} \sqrt{1-x} d x=\frac{\lambda \delta_{m}^{n+1}}{n+1} \cdot \frac{2 \dot{\delta}^{(-)}}{3}
\end{aligned}
$$

On the other hand, as for the similar dynamic equation as illustrated in Eq. (10), the work done by the dissipative force during the compression phase is

$$
\Delta E_{c}^{*}=\int_{0}^{\delta_{m}} \lambda \delta^{n} \widehat{\dot{\delta}}_{c} d \delta=\frac{\lambda \delta_{m}^{n+1}}{n+1} \widehat{\dot{\delta}}_{c}
$$

Combining Eqs. (14) and (15), the equivalent velocity during the compression phase can be deduced:

$$
\widehat{\dot{\delta}}_{c}=\frac{2 \dot{\delta}^{(-)}}{3}
$$

Similar to the derivation for the compression phase, by integrating Eq. (10) over the restitution phase, we obtain

$$
\left(c_{r} \dot{\delta}^{(-)}\right)^{2}-\dot{\delta}^{2}=\frac{2\left(k+\lambda \hat{\dot{\delta}}_{r}\right)}{m(n+1)} \delta^{n+1}
$$

Substituting $\dot{\delta}=0$ into Eq. (17), it can be deduced that

$$
\delta_{m}^{n+1}=\frac{m(n+1)}{2\left(k+\lambda \widehat{\dot{\delta}}_{r}\right)}\left(c_{r} \dot{\delta}^{(-)}\right)^{2}
$$

Noting that the velocities have opposite directions during the restitution phase and compression phase, the function between the deformation velocity $\dot{\delta}$ and indentation depth $\delta$ during the restitution phase can be derived:

$$
\dot{\delta}=-c_{r} \dot{\delta}^{(-)} \sqrt{1-\left(\frac{\delta}{\delta_{m}}\right)^{n+1}}
$$

The work done by the dissipative force during the restitution phase is

$$
\Delta \mathrm{E}_{r}=\int_{\delta_{m}}^{0} \lambda \delta^{n} \dot{\delta} d \delta=-\int_{\delta_{m}}^{0} \lambda \delta^{n} c_{r} \dot{\delta}^{(-)} \sqrt{1-\left(\frac{\delta}{\delta_{m}}\right)^{n+1}} d \delta=\frac{\lambda \delta_{m}^{n+1}}{n+1} \cdot \frac{2 c_{r} \dot{\delta}^{(-)}}{3}
$$

For the similar dynamic equation described in Eq. (10), the work done by the dissipative force during the restitution phase can be gained,

$$
\Delta E_{r}^{*}=\int_{\delta_{m}}^{0} \lambda \delta^{n} \widehat{\dot{\delta}}_{r} d \delta=-\frac{\lambda \delta_{m}^{n+1}}{n+1} \widehat{\dot{\delta}}_{r}
$$

Combining Eqs. (20) and (21), the equivalent velocity of the restitution phase can be expressed as

$$
\widehat{\dot{\delta}}_{r}=\frac{-2 c_{r} \dot{\delta}^{(-)}}{3}
$$

In summary, the approximate dynamic equation can be described as follows:

$$
\begin{cases}m \ddot{\delta}+\left(k+\lambda \frac{2 \dot{\delta}^{(-)}}{3}\right) \delta^{n}=0 & \text { Compression phase } \\ m \ddot{\delta}+\left(k-\lambda \frac{2 c_{r} \dot{\delta}^{(-)}}{3}\right) \delta^{n}=0 & \text { Restitution phase }\end{cases}
$$

The above discussion suggests that the approximate dynamic equation with properly chosen velocities can accurately approximate the system dynamic equation. There will be more discussion of this aspect in the following sections.

Furthermore, based on the approximate dynamic equation, the relation $t(\delta)$ can be determined analytically in closed form in terms of beta functions. 
Let us consider the compression phase, Eq. (13) can be rewritten as

$$
d t=\frac{d \delta}{\dot{\delta}(-) \sqrt{1-\left(\frac{\delta}{\delta_{m}}\right)^{n+1}}}=\frac{\delta_{m}}{\dot{\delta}(-) \sqrt{1-\left(\frac{\delta}{\delta_{m}}\right)^{n+1}}} d\left(\frac{\delta}{\delta_{m}}\right)
$$

Let us put $\vartheta=\left(\frac{\delta}{\delta_{m}}\right)^{n+1}$, it can be deduced that

$$
\frac{1}{n+1} \vartheta^{\frac{1}{n+1}-1} d \vartheta=d\left(\frac{\delta}{\delta_{m}}\right)
$$

Substituting $\vartheta=\left(\frac{\delta}{\delta_{m}}\right)^{n+1}$ and Eq. (25) into Eq. (24), we obtain

$$
d t=\frac{\delta_{m}}{\dot{\delta}(-)(n+1)}(1-\vartheta)^{-\frac{1}{2}} \vartheta^{\frac{1}{n+1}-1} d x
$$

The integral of Eq. (26) can be expressed in terms of the incomplete beta function, as shown in Eq. (27)

$$
\begin{aligned}
t & =\int_{0}^{\xi} \frac{\delta_{m}}{\dot{\delta}(-)(n+1)}(1-\vartheta)^{-\frac{1}{2}} \vartheta^{\frac{1}{n+1}-1} d x \\
& =\frac{\delta_{m}}{\dot{\delta}(-)(n+1)} \operatorname{Beta}\left(\xi ; \frac{1}{n+1}, \frac{1}{2}\right)
\end{aligned}
$$

where $\xi=\frac{\delta_{t}}{\delta_{m}}<1, \delta_{t}$ is the indentation deformation at time $t$. Substituting Eqs. (12) and (16) into Eq. (27), the contact time during the compression phase can be derived

$$
t=\int_{0}^{\xi} \frac{\left(\frac{m(n+1)}{2\left(k+\frac{2 \lambda \dot{\delta}(-)}{3}\right)} \dot{\delta}^{(-) 2}\right)^{\frac{1}{n+1}}}{\dot{\delta}^{(-)}(n+1)}(1-\vartheta)^{-\frac{1}{2}} \vartheta^{\frac{1}{n+1}-1} d x
$$

As $\xi=1$, the time of the whole compression phase can be obtained in terms of beta function

$$
t_{c}=\frac{\delta_{m}}{\dot{\delta}^{(-)}(n+1)} \operatorname{Beta}\left(\frac{1}{n+1}, \frac{1}{2}\right)
$$

Similar to the derivation for the compression phase, the relation $t(\delta)$ over the restitution phase can be described as

$$
t=\frac{\delta_{m}}{c_{r} \dot{\delta}^{(-)}(n+1)} \operatorname{Beta}\left(\xi ; \frac{1}{n+1}, \frac{1}{2}\right)
$$

The time of the whole restitution phase can be deduced,

$$
t_{r}=\frac{\delta_{m}}{c_{r} \dot{\delta}^{(-)}(n+1)} \operatorname{Beta}\left(\frac{1}{n+1}, \frac{1}{2}\right)
$$

Therefore, the time of the whole contact phase can be obtained in terms of the beta function

$$
T=t_{c}+t_{r}=\left(1+\frac{1}{c_{r}}\right) \frac{\delta_{m}}{\dot{\delta}^{(-)}(n+1)} \operatorname{Beta}\left(\frac{1}{n+1}, \frac{1}{2}\right)
$$

\subsection{The expression of the hysteresis damping factor}

Based on the approximate dynamic equation, the expression of contact force can be proposed for contact-impact problems with complex geometries and the exponent $n$ for the system dynamic equation is arbitrary.

Combined with Eqs. (14) and (20), we have

$$
\frac{\Delta \mathrm{E}_{c}}{\Delta \mathrm{E}_{r}}=\frac{1}{c_{r}}
$$

Due to $\Delta E_{\text {loss }}=\Delta \mathrm{E}_{c}+\Delta \mathrm{E}_{r}$, substituting Eq. (33) into Eq. (7) yields

$$
\Delta \mathrm{E}_{c}=\frac{m \dot{\delta}^{(-) 2}\left(1-c_{r}\right)}{2}
$$

According to energy balance and the law of conservation of linear momentum in the period from $t^{(-)}$to $t_{m}$, we can obtain the following equations:

$$
\left\{\begin{array}{l}
\frac{m_{i}\left(v_{i}^{-}\right)^{2}}{2}+\frac{m_{j}\left(v_{j}^{-}\right)^{2}}{2}=\frac{\left(m_{i}+m_{j}\right) v_{i j}^{2}}{2}+\Delta E_{k}+\Delta E_{c} \\
m_{i} v_{i}^{-}+m_{j} v_{j}^{-}=\left(m_{i}+m_{j}\right) v_{i j}
\end{array}\right.
$$


$\Delta E_{k}$ is the elastic potential energy stored from $\mathrm{t}^{(-)}$to $\mathrm{t}_{\mathrm{m}}$ and can be calculated as

$$
\Delta E_{k}=\int_{0}^{\delta_{m}} k \delta^{n} d \delta=\frac{k \delta_{m}^{n+1}}{n+1}
$$

Combining Eqs. (34)-(36), by denoting the equivalent mass $m=\frac{m_{i} m_{j}}{m_{i}+m_{j}}$ and $\dot{\delta}^{(-)}=v_{i}^{-}-v_{j}^{-}$, it can be deduced that,

$$
\frac{m \dot{\delta}^{(-) 2}}{2}=\frac{k \delta_{m}^{n+1}}{n+1}+\frac{m \dot{\delta}^{(-) 2}\left(1-c_{r}\right)}{2}
$$

which yields

$$
\delta_{m}^{n+1}=\frac{c_{r} m(n+1) \dot{\delta}^{(-) 2}}{2 \mathrm{k}}
$$

Substituting Eq. (38) into Eqs. (14) and (20), we obtain

$$
\begin{aligned}
\Delta \mathrm{E}_{c} & =\frac{\lambda c_{r} m \dot{\delta}^{(-) 3}}{3 k} \\
\Delta \mathrm{E}_{r} & =\frac{\lambda c_{r}^{2} m \dot{\delta}^{(-) 3}}{3 k}
\end{aligned}
$$

Combining Eqs. (7), (39) and (40), we can deduce that

$$
\frac{\lambda c_{r} m \dot{\delta}^{(-) 3}}{3 k}+\frac{\lambda c_{r}^{2} m \dot{\delta}^{(-) 3}}{3 k}=\frac{m\left(1-c_{r}^{2}\right) \dot{\delta}^{(-) 2}}{2}
$$

Then, the description of the hysteresis damping factor can be obtained:

$$
\lambda=\frac{3 k\left(1-c_{r}\right)}{2 c_{r} \dot{\delta}^{(-)}}
$$

This description is the same as the model provided in [12], which considered $n=1.5$ for the system dynamic equation.

Combining Eqs. (15), (34) and (38), the description of the hysteresis damping factor with respect to the equivalent velocity during the compression phase can be deduced:

$$
\lambda=\frac{k\left(1-c_{r}\right)}{c_{r} \hat{\dot{\delta}}_{c}}=\frac{3 k\left(1-c_{r}\right)}{2 c_{r} \dot{\delta}^{(-)}}
$$

It reveals that Eq. (43) is completely consistent with Eq. (42), and the form of Eq. (43) is convenient for the model modification; the details will be discussed in Section 4.4.

Substituting Eqs. (16), (22) and (42) into Eqs. (12) and (18), which are derived from the approximate dynamic equation, respectively, we obtain

$$
\begin{aligned}
& \delta_{m}^{n+1}=\frac{m(n+1)\left(\dot{\delta}^{(-)}\right)^{2}}{2\left(k+\frac{3 k\left(1-c_{r}\right)}{2 c_{r} \dot{\delta}(-)} \cdot \frac{2 \dot{\delta}(-)}{3}\right)}=\frac{c_{r} m(n+1) \dot{\delta}^{(-) 2}}{2 k} \\
& \delta_{m}^{n+1}=\frac{m(n+1)\left(c_{r} \dot{\delta}^{(-)}\right)^{2}}{2\left(k-\frac{3 k\left(1-c_{r}\right)}{2 c_{r} \dot{\delta}^{(-)}} \cdot \frac{2 c_{r} \dot{\delta}^{(-)}}{3}\right)}=\frac{c_{r} m(n+1) \dot{\delta}^{(-) 2}}{2 k}
\end{aligned}
$$

It can be seen that Eqs. (44) and (45) are completely consistent with Eq. (38), which is derived according to energy balance and the law of conservation of linear momentum. The feasibility of establishing a new model based on the approximate dynamic equation is further confirmed.

\subsection{Establishing the new model via modification}

Although the derivations in Sections 4.2 and 4.3 demonstrate that the approximate dynamic equation achieves a good approximation of the system dynamic equation in terms of the system energy consumption and the maximum indentation depth, it is worth discussing the accuracy of the approximate of the system dynamic equation via the approximate equation in the contact-impact process.

A series of numerical experiments is conducted to evaluate the accuracy of the approximate dynamic equation in representing the dynamic behavior of the system, as shown in Figs. 3 and 4. The simulation results from Eq. (8) are deemed accurate, and the results from Eq. (23) are deemed approximate. The damping coefficient is calculated by using Eq. (43). The generalized stiffness parameter $k$ equals $2.41 \mathrm{E} 11 \mathrm{pa}$, and $\dot{\delta}^{(-)}$equals $2.895 \mathrm{~m} / \mathrm{s}$. It should be pointed out that all numerical 


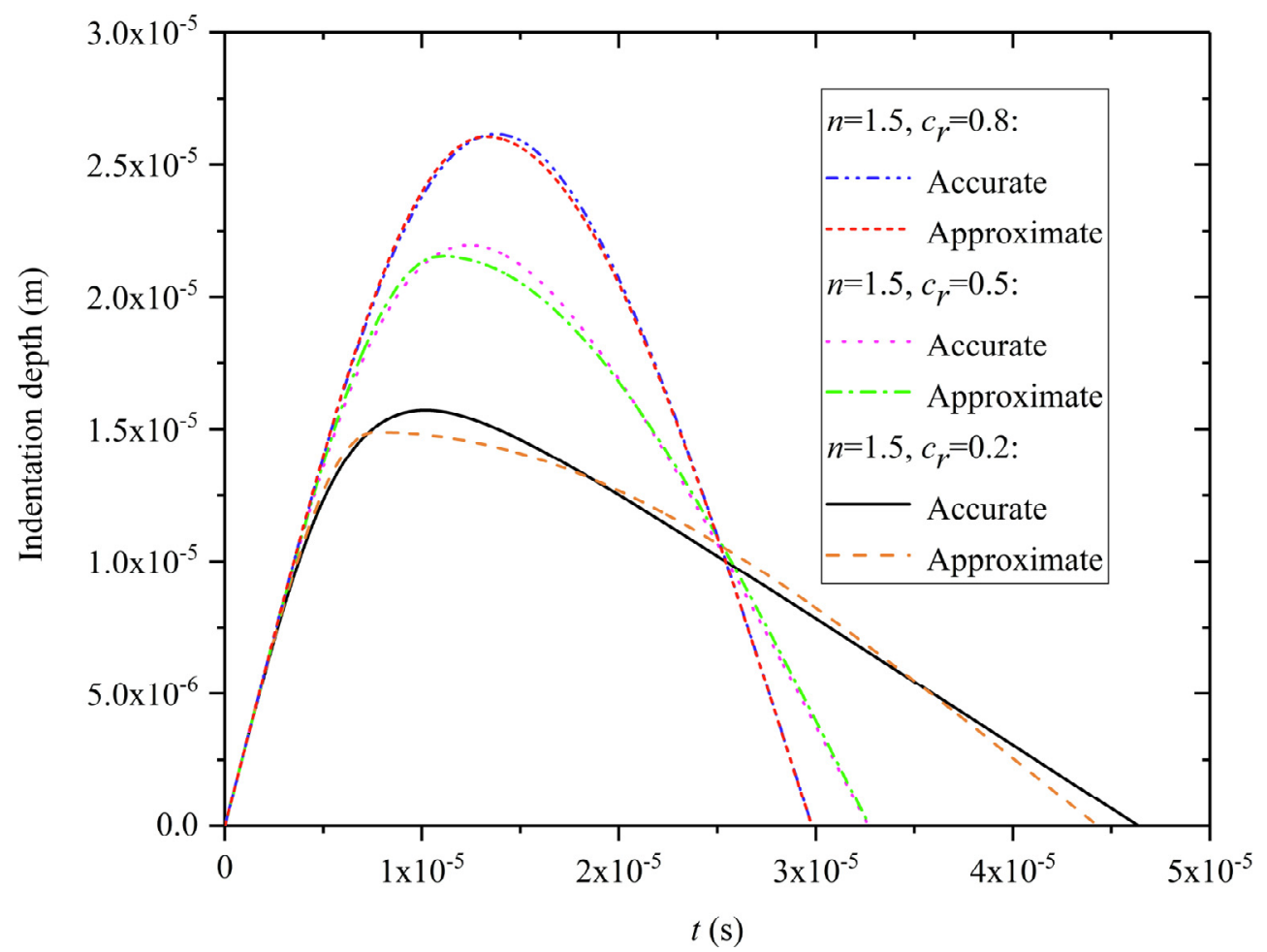

Fig. 3. Dynamic behaviors of the system dynamic equation and the approximate dynamic equation with $n=1.5$.

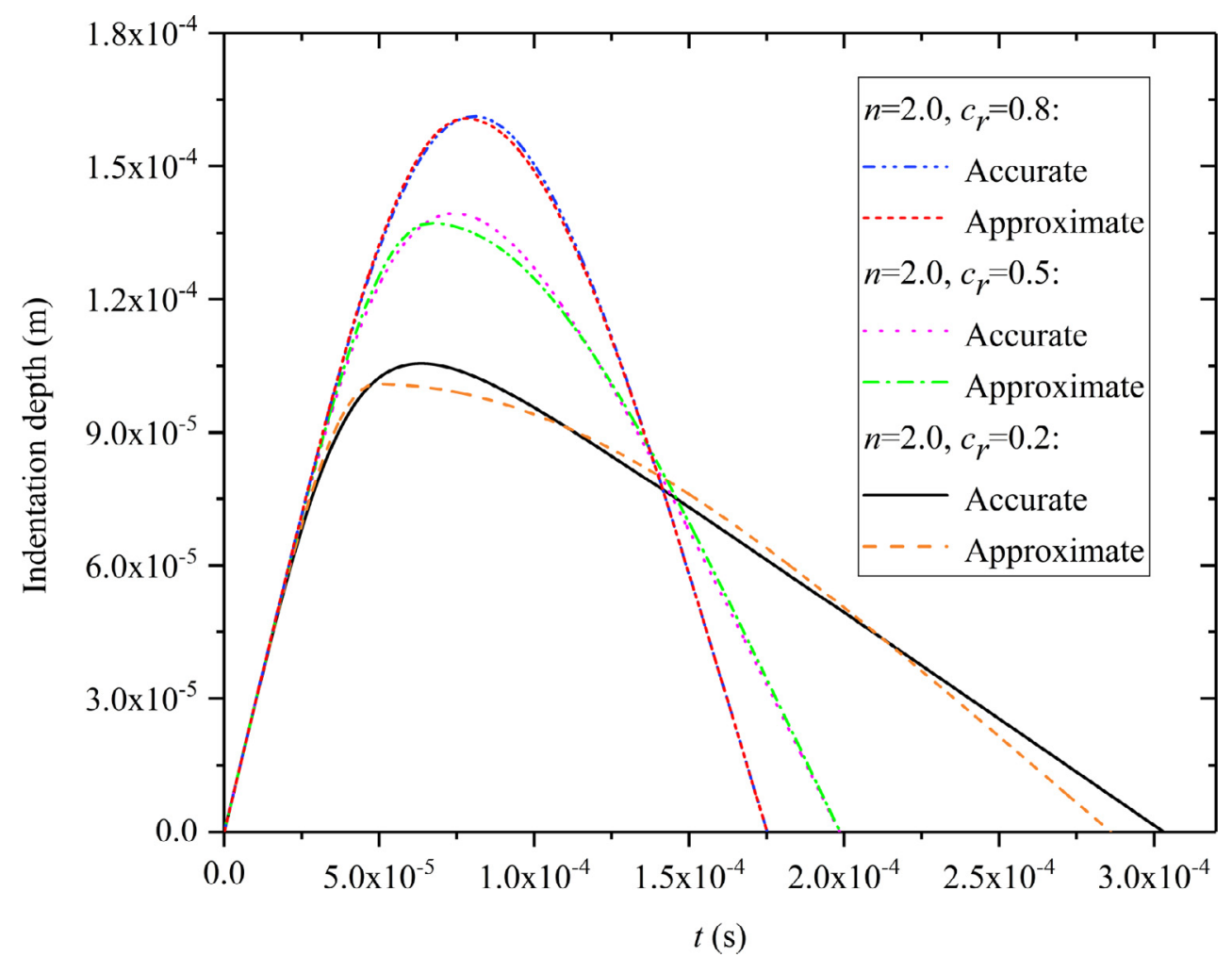

Fig. 4. Dynamic behaviors of the system dynamic equation and the approximate dynamic equation with $n=2.0$. 
simulations in this paper are conducted by utilizing MATLAB codes and the four-order-Runge-Kutta method, and the time steps are set as $1 \mathrm{E}-8 \mathrm{~s}$.

It can be seen that the approximate equation does not perfectly match the system dynamics equation. There is a deviation in the description of the system dynamic behavior between the approximate dynamic equation and the system dynamic equation, and the smaller the coefficient of restitution $c_{r}$, the larger the deviation is. Naturally, we can speculate that it is possible to obtain a more accurate model by modifying the formulas, which are obtained based on the approximate equation, with minor adjustments of the equivalent velocity, as shown in Eq. (43).

The modification is based on nondimensional analysis, and we introduce the nondimensional variables $x=\delta / \delta_{m}$ and $\tau=$ $t /\left(\delta_{m} / \dot{\delta}^{(-)}\right)[32]$, it can be deduced that

$$
\begin{aligned}
& \frac{d \delta}{d t}=\dot{\delta}^{(-)} \frac{d x}{d \tau} \\
& \frac{d^{2} \delta}{d t^{2}}=\frac{\left(\dot{\delta}^{(-)}\right)^{2}}{\delta_{m}} \frac{d^{2} x}{d \tau^{2}}
\end{aligned}
$$

Substituting $x=\delta / \delta_{m}$, by placing Eqs. (46) and (47) into the system dynamic equation as shown in Eq. (8), we obtain

$$
\frac{m\left(\dot{\delta}^{(-)}\right)^{2}}{\delta_{m}} \frac{d^{2} x}{d \tau^{2}}+k \delta_{m}^{n} x^{n}+\lambda \delta_{m}^{n} x^{n} \dot{\delta}^{(-)} \frac{d x}{d \tau}=0
$$

The following nondimensional form can be obtained:

$$
\frac{d^{2} x}{d \tau^{2}}+k^{*} x^{n}+\lambda^{*} x^{n} \frac{d x}{d \tau}=0
$$

The relevant nondimensional parameters $k^{*}$ and $\lambda^{*}$ are expressed as

$$
\begin{aligned}
k^{*} & =\frac{k \delta_{m}^{n+1}}{m\left(\dot{\delta}^{(-)}\right)^{2}} \\
\lambda^{*} & =\frac{\lambda \delta_{m}^{n+1}}{m \dot{\delta}^{(-)}}
\end{aligned}
$$

Substituting Eq. (44) into Eqs. (50) and (51), we obtain

$$
\begin{aligned}
k^{*} & =\frac{c_{r}(n+1)}{2} \\
\lambda^{*} & =\frac{\lambda c_{r}(n+1) \dot{\delta}^{(-)}}{2 k}
\end{aligned}
$$

Eq. (43), which is obtained based on the approximate equation, can be modified by a minor reduction of the equivalent velocity $\widehat{\dot{\delta}}_{c}$ to obtain

$$
\lambda=\frac{k\left(1-c_{r}\right)}{\alpha c_{r} \hat{\delta}_{c}}=\frac{3 k\left(1-c_{r}\right)}{2 \alpha c_{r} \dot{\delta}^{(-)}}
$$

where $\alpha$ is the modification parameter representing the minor reduction of the equivalent velocity.

Substituting Eq. (54) into Eq. (53), we obtain

$$
\lambda^{*}=\frac{3\left(1-c_{r}\right)(n+1)}{4 \alpha}
$$

The nondimensional parameters $k^{*}$ and $\lambda^{*}$ depend only on the coefficient of restitution $c_{r}$ and the exponent $n$ which represents the topological properties of the contacting surfaces. Eq. (49) can be solved numerically with the preset values of $n$ and pre-restitution coefficient $c_{r}$, and then the post-restitution coefficient can be obtained from the velocity at the end of the collision.

For continuous contact force models, previous studies have shown that the pre-restitution coefficient differs from the post-restitution coefficient, which should theoretically be the same [1,10,12,34]. As the pre-restitution coefficient is obtained via a physical experiment, the deviation of the post-restitution coefficient from the pre-restitution coefficient represents the deviation of the simulation results of velocity from the actual values of velocity and the deviation of the output predicted by the model from the actual behavior of the system. Therefore, the deviation of the post-restitution coefficient from the pre-restitution coefficient is considered an important criterion for modifying the model [34].

A series of numerical simulations are conducted with the initial value of the normalized velocity $\frac{d x}{d \tau}$ equals to 1 and $n=1.5$; the optimum values of the modification parameter $\alpha$ for different values of restitution coefficient $c_{r}$ are established 


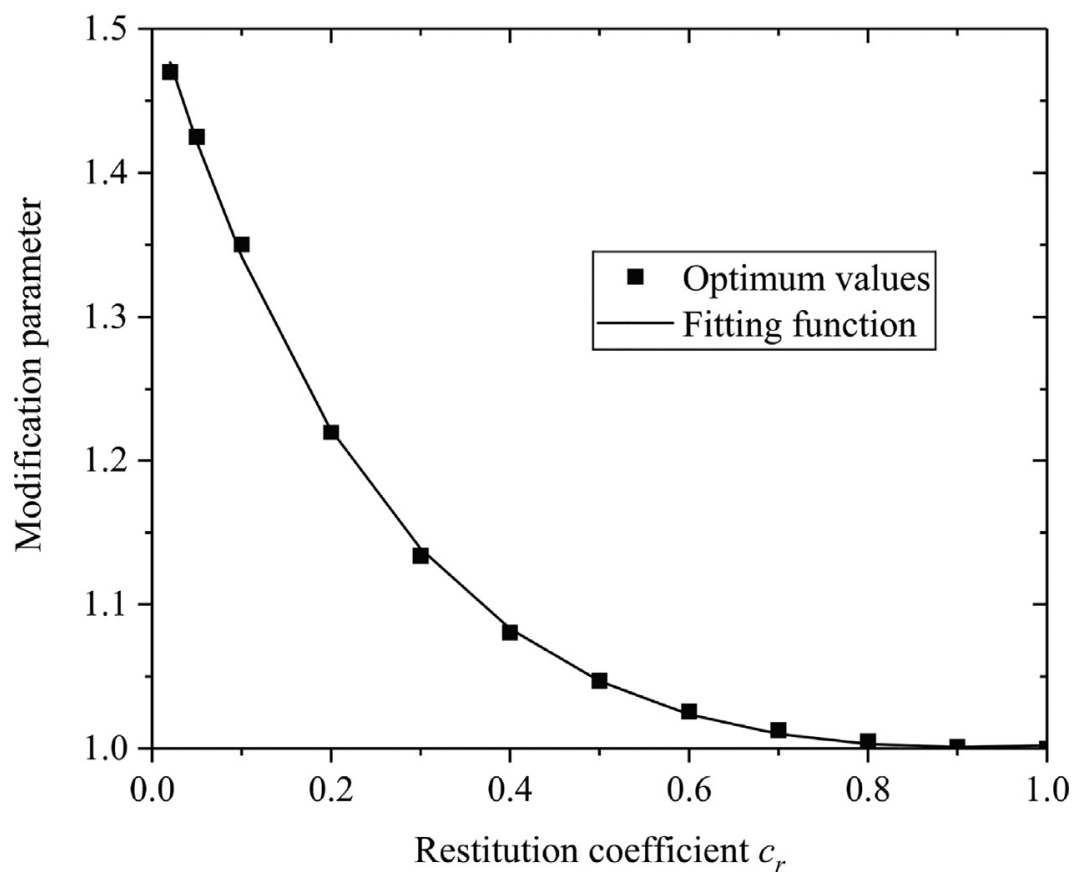

Fig. 5. The optimum values and fitting curve of modification parameter $\alpha$ for different values of restitution coefficient $c_{r}$ as $n=1.5$.

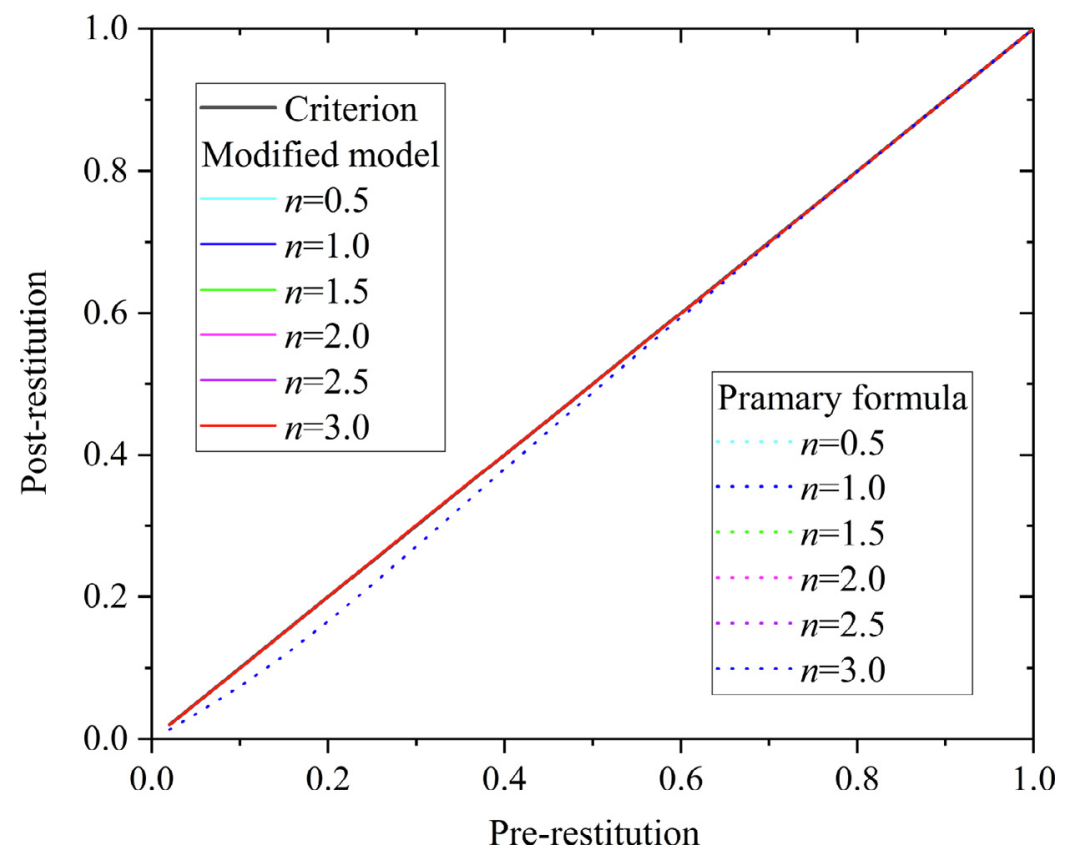

Fig. 6. Relation between the post- and pre-restitution coefficients for different values of $n$.

based on the trial calculation method, and an cubic curve fitting is conducted, as shown in Fig. 5. The expression of the fitting function is as follows.

$$
\alpha=0.6181 e^{-3.52 c_{r}}+0.899 e^{0.09025 c_{r}}
$$

Then, the numerical simulations are conducted based on Eqs. (49), (52), (55) and (56) with different values of $n$; the plots of the post vs. pre-restitution coefficient for different values of $n$ are given in Fig. 6 . It can be seen that the effects of the values of $\mathrm{n}$ on the modified parameter $\alpha$ are negligible; therefor, Eq. (56) can be treated as the optimum function of the modification parameter $\alpha$ for different values of $n$. 
Table 1

Compliant continuous contact models.

\begin{tabular}{|c|c|c|c|}
\hline Model & $\mathrm{n}$ & $\beta$ & $\lambda$ \\
\hline Hunt and Crossley [27] & arbitrary $n$ & $\beta=n$ & $\frac{3\left(1-c_{r}\right)}{2} \frac{k}{\delta(-)}$ \\
\hline Lankarani and Nikravesh [21] & arbitrary $n$ & $\beta=n$ & $\frac{3\left(1-c_{r}^{2}\right)}{4} \frac{k}{\delta(-)}$ \\
\hline Kuwabara and Kono [32] (for $\left.\mathrm{c}_{\mathrm{r}} \approx 1\right)$ & 1.5 & 0.5 & $\frac{\left(1-c_{r}\right)}{1.009}\left(\frac{\dot{\delta}(-)}{m^{2}\left(4 k_{/ 5}\right)^{3}}\right)^{-\frac{1}{5}}$ \\
\hline Flores et al. [10] & 1.5 & 1.5 & $\frac{8\left(1-c_{r}\right)}{5 c_{r}} \frac{k}{\delta(-)}$ \\
\hline Gharib and Hurmuzlu [33] & arbitrary $n$ & $\beta=n$ & $\frac{1}{c_{r}} \frac{k}{\delta(-)}$ \\
\hline Hu and Guo $[12]$ & 1.5 & 1.5 & $\frac{3\left(1-c_{r}\right)}{2 c_{r}} \frac{k}{\delta(-)}$ \\
\hline Shen et al. [34] (without external forces) & 1.5 & 1.5 & $\frac{3\left(1-c_{r}\right)}{2 c^{0.89}} \frac{\delta}{\delta(-)}$ \\
\hline The model in this paper & arbitrary $n$ & $\beta=n$ & Eq. (57) \\
\hline
\end{tabular}

The description of the modified hysteresis damping factor in the new model can be obtained based on Eq. (43) and (56):

$$
\lambda=\frac{3 k\left(1-c_{r}\right)}{2\left(0.6181 e^{-3.52 c_{r}}+0.899 e^{0.09025 c_{r}}\right) c_{r} \dot{\delta}^{(-)}}
$$

It can be noted that for the purely elastic contact, the hysteresis damping factor $\lambda$ equals zero. Correspondingly, the contact force in the new model can be expressed as

$$
F_{N}=k \delta^{n}+\frac{3 k\left(1-c_{r}\right) \delta^{n} \dot{\delta}}{2\left(0.6181 e^{-3.52 c_{r}}+0.899 e^{0.09025 c_{r}}\right) c_{r} \dot{\delta}^{(-)}}
$$

Substituting Eq. (54) into Eq. (23), the approximate dynamic equation can be modified as:

$$
\left\{\begin{aligned}
m \ddot{\delta}+\left(\frac{1+\alpha c_{r}-c_{r}}{\alpha \alpha_{r}}\right) k \delta^{n}=0 & \text { Compression phase } \\
m \ddot{\delta}+\left(\frac{\alpha+c_{r}-1}{\alpha}\right) k \delta^{n}=0 & \text { Restitution phase }
\end{aligned}\right.
$$

The new contact-impact model described in Eqs. (57) and (58) is valid for direct central and frictionless impacts with complex geometries, as the exponent $n$ in the system dynamic equation that characterizes the topological properties of the contacting surfaces is more complicated.

\section{Validation and comparison of the new model}

In this section, the new contact-impact model described in Eqs. (57) and (58) is verified through the comparison with published experimental data and the analysis of simulation data. The validation and comparison are carried out from the following aspects: consistency of post- and pre-restitution coefficients, contact duration time $T$ and peak contact force $F_{\max }$, which are crucial for the description of the contact-impact behavior of a multibody system [9]. Seven different compliant continuous contact models are utilized for the validation and comparison of the new model, including the models developed by Hunt and Crossley [27], Lankarani and Nikravesh [21], Kuwabara and Kono [32], Flores et al. [10], Gharib and Hurmuzlu [33], Hu and Guo [12], and Shen et al. [34]. In these models, the contact forces, which are composed of an elastic force and a dissipative force, can be written in a common form [9]:

$$
F_{N}=k \delta^{n}+\lambda \delta^{\beta} \dot{\delta}
$$

where $n$ is the exponent of the indentation depth which depends on the geometry of the contacting surfaces, and $\beta$ is the exponent associated with damping. Table 1 presents an overview of these models.

It should be noted that the expression given in Table 1 is a special case of Kuwabara and Kono model, which is derived under the assumption that $c_{r} \approx 1$ [32]; thus, the functional relationship between the hysteresis damping factor $\lambda$ and coefficient of restitution $c_{r}$ can be established. The expression of Shen et al. model presented in Table 1 is obtained by eliminating the influence of external forces [34]. And it should be noticed that the Gharib and Hurmuzlu model is developed for a low coefficient of restitution impact [33].

\subsection{Experimental validation and comparison}

In this section, experimental results [49] of the direct central impact between a steel ball and a cylindrical specimen are utilized to validate and compare the contact models listed in Table 1. Fig. 7 shows a sketch of the experimental setup. A pendulum test rig is utilized for the impact force experiments, as shown in Fig. 7a. The peak impact force $F_{\max }$, impact duration time $T$ and initial impact velocity $\dot{\delta}^{(-)}$of the experiments can be found in Zhang and Sharf [49], while the coefficient of restitution associated with certain initial velocity is measured by the dropweight tower experiment, as shown in Fig. 7b [49]. Two different materials for the cylindrical specimens (C1 and C2) with different yield strengths are selected in [49]. The impact experimental results of specimen $\mathrm{C} 2$ are more comprehensive and include the restitution coefficient, duration 


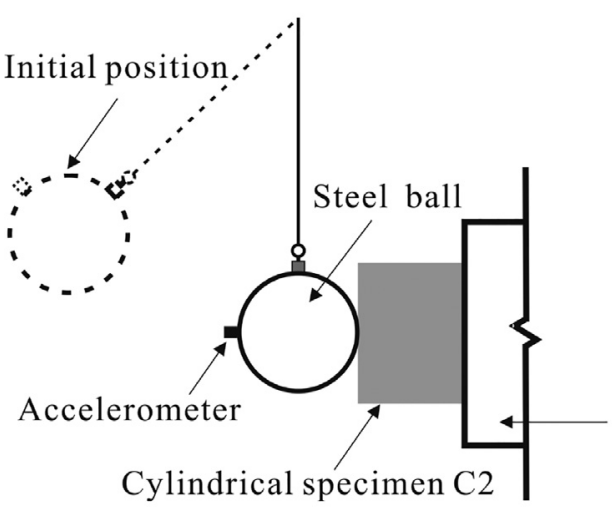

(a)
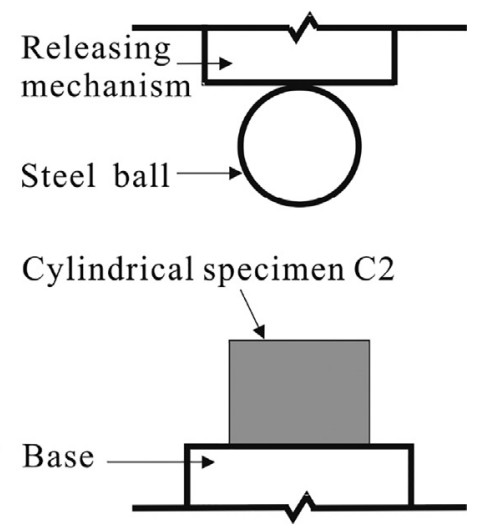

(b)

Fig. 7. The sketch of the experimental setup: (a) pendulum test rig, (b) dropweight tower, (Adapted from [49]).

Table 2

Comparison of the simulation and experimental results for specimen $\mathrm{C} 2$ with an initial impact velocity of $0.15 \mathrm{~m} / \mathrm{s}$.

\begin{tabular}{|c|c|c|c|c|c|c|}
\hline & $c_{r}$ & Relative error [\%] & $T(10 \mathrm{E}-4 \mathrm{~s})$ & Relative error [\%] & $F_{\max }(N)$ & Relative error [\%] \\
\hline Experiment results [49] & 0.8892 & & 2.52 & & 1076.6 & \\
\hline Hunt and Crossley [27] & 0.9002 & 1.23 & 2.614 & 3.71 & 1095.0 & 1.71 \\
\hline Lankarani and Nikravesh [21] & 0.9052 & 1.80 & 2.612 & 3.65 & 1097.4 & 1.93 \\
\hline Kuwabara and Kono [32] & 1.0000 & 12.46 & 2.584 & 2.53 & 1153.5 & 7.14 \\
\hline Flores et al. [10] & 0.8825 & -0.75 & 2.620 & 3.96 & 1086.9 & 0.96 \\
\hline Gharib and Hurmuzlu [33] & 0.5637 & -36.60 & 2.820 & 11.89 & 1054.0 & -2.10 \\
\hline $\mathrm{Hu}$ and Guo [12] & 0.8891 & -0.02 & 2.618 & 3.87 & 1089.8 & 1.23 \\
\hline Shen et al. [34] & 0.8903 & 0.13 & 2.617 & 3.85 & 1090.4 & 1.28 \\
\hline The model in this paper & 0.8892 & -0.00 & 2.618 & 3.87 & 1089.9 & 1.23 \\
\hline
\end{tabular}

Table 3

Comparison of the simulation and experimental results for specimen $\mathrm{C} 2$ with an initial impact velocity of $0.5 \mathrm{~m} / \mathrm{s}$.

\begin{tabular}{|c|c|c|c|c|c|c|}
\hline & $c_{r}$ & Relative error [\%] & $T(10 \mathrm{E}-4 \mathrm{~s})$ & Relative error [\%] & $F_{\max }(N)$ & Relative error [\%] \\
\hline Experiment results [49] & 0.7568 & & 2.07 & & 4364.6 & \\
\hline Hunt and Crossley [27] & 0.8036 & 6.19 & 2.085 & 0.72 & 4491.3 & 2.90 \\
\hline Lankarani and Nikravesh [21] & 0.8234 & 8.80 & 2.078 & 0.38 & 4515.5 & 3.46 \\
\hline Kuwabara and Kono [32] & 1.0000 & 32.14 & 2.031 & -1.89 & 4891.8 & 12.08 \\
\hline Flores et al. [10] & 0.7431 & -1.82 & 2.109 & 1.88 & 4439.0 & 1.70 \\
\hline Gharib and Hurmuzlu [33] & 0.5215 & -31.09 & 2.253 & 8.84 & 4517.3 & 3.50 \\
\hline $\mathrm{Hu}$ and Guo [12] & 0.7554 & -1.90 & 2.104 & 1.63 & 4447.0 & 1.89 \\
\hline Shen et al. [34] & 0.7611 & 0.56 & 2.101 & 1.51 & 4451.1 & 1.98 \\
\hline The model of this paper & 0.7564 & -0.05 & 2.103 & 1.60 & 4447.7 & 1.90 \\
\hline
\end{tabular}

time and peak impact force, so the comparison of this paper is conducted with the experimental data for specimen $\mathrm{C} 2$. The basic parameters of the experiment can be found in [49]: the mass of the steel ball is $0.54 \mathrm{~kg}$, the power exponent $n=1.5$ and the stiffness coefficient $k=2.4144 \mathrm{E} 10 \mathrm{~N} / \mathrm{s}^{n}$. A more detailed description of the experiment is given in [49]. Corresponding numerical simulations with different initial impact velocities are carried out, and the validation and comparison of the contact models are conducted from the consistency of the post- and pre-restitution coefficients, the contact duration time and the value of the peak contact force. The experimental and simulation results are presented in Tables 2 and 3 , as well as the relative percentage errors.

As shown in Tables 2 and 3, the initial impact velocities are $0.15 \mathrm{~m} / \mathrm{s}$ and $0.5 \mathrm{~m} / \mathrm{s}$, and the corresponding pre-restitution coefficients are 0.8892 and 0.7567 . This reveals that the models considered in this analysis, except the Kuwabara and Kono model [32] and Gharib and Hurmuzlu model [33], exhibit good consistencies with experimental results, especially the ones proposed by Flores et al., Hu and Guo, Shen et al. and this research, the relative errors of these models are less than $\pm 4 \%$. Because the expressions of Kuwabara and Kono model and Gharib and Hurmuzlu model presented in Table 1 are developed for $c_{r} \approx 1$ and a low coefficient of restitution, respectively, it is not difficult to understand that the large deviation between the simulation results and the experimental data in Tables 2 and 3. The small deviations between the simulation results of the new model and the experimental data can be observed, which supports the validity and potentiality of the new model. Especially, the new model provides the best accuracy on the consistency of post- and pre-restitution coefficients relative to those of the other models. 


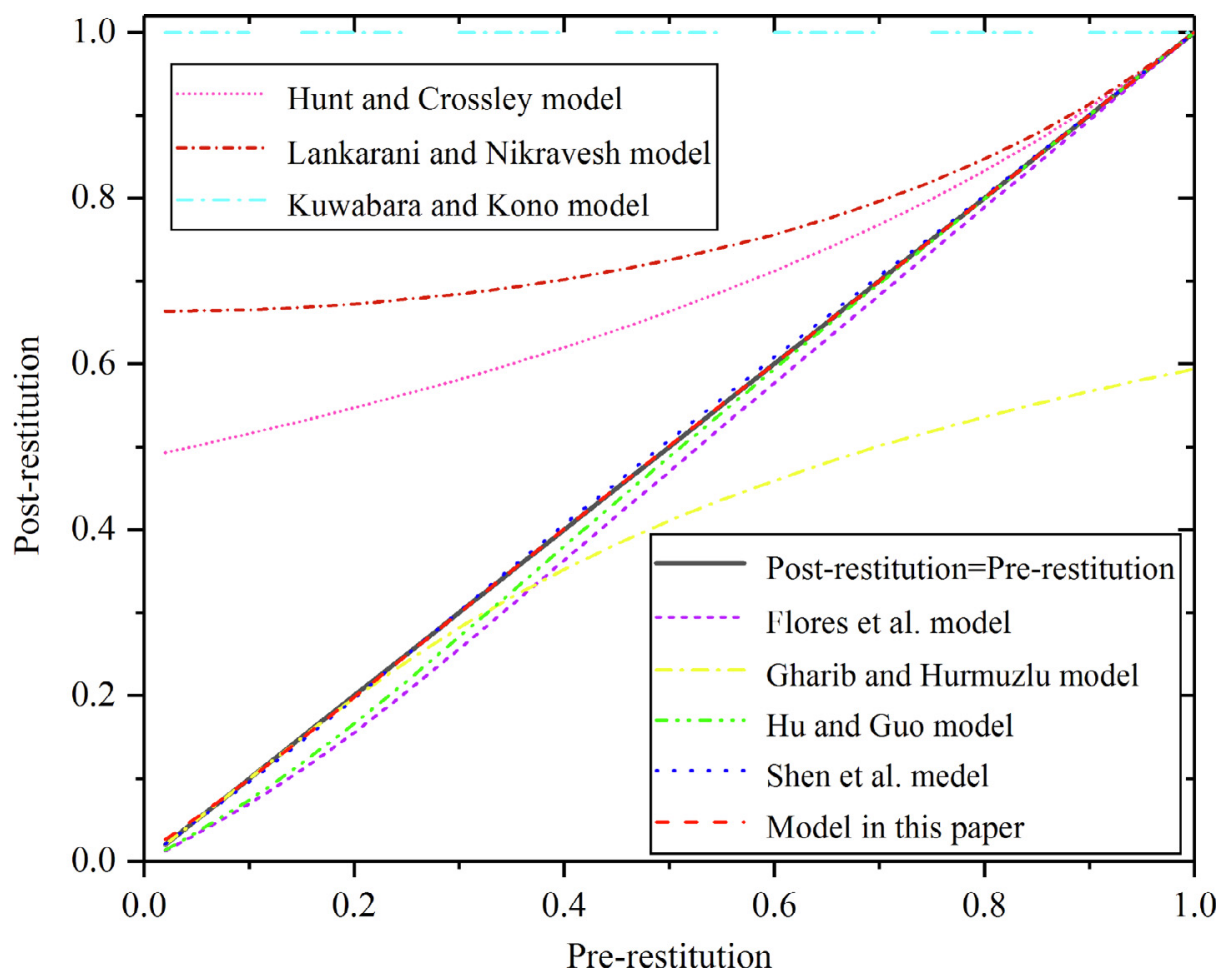

Fig. 8. Relation between the post- and pre-restitution coefficients for different contact models as $n=1.5$.

\subsection{Validation and comparison in terms of the consistency of post-and pre-restitution coefficients}

In this section, a series of numerical simulations are conducted to validate and compare the contact models in terms of the consistency of post- and pre-restitution coefficients over the entire range of restitution coefficients. The basic parameters of the simulations are as the same as those in Section 5.1. From a physical point of view, the smaller the difference between the post- and pre-restitution coefficients, the more closely the collision process described by the model matches the actual situation [12].

The plots of the post vs. pre-restitution coefficient for different contact force models as $n=1.5$ are given in Fig. 8 . It can be seen that the Lankarani and Nikravesh model and the Hunt and Crossley model are suitable for high values of restitution coefficient, Gharib and Hurmuzlu model provides a good performance for the low values of the restitution coefficient. The models proposed by Flores et al., Hu and Guo, Shen et al. and this research have better performances relative to other models for the post-restitution coefficient vs. pre-restitution coefficient over the entire range of restitution coefficients, especially the Shen et al. model and the new model presented in this paper.

Additionally, numerical simulations with different values of $n$ are conducted, the exponent $n$, which describes the topological properties of the contacting surfaces, is set to 0.5, 1.0, 2.0 and 3.0. As shown in Table 1, four models are available for the contact problems with arbitrary values of $n$ : Hunt and Crossley model [27], Lankarani and Nikravesh model [21], Gharib and Hurmuzlu model [33] and the model described in this paper. The plots of the post vs. pre-restitution coefficient are illustrated in Fig. 9. It can be seen that the new model provides the best fit for the post-restitution coefficient vs. pre-restitution coefficient relative to other models over the entire range of restitution coefficients.

Therefore, the capability of the new model is validated and compared with published experimental data and the numerical results of eight different contact models. The new model shows high accuracy on the consistency of post- and pre-restitution coefficient, contact duration time and peak contact force, and it is available for the contact problems with complex geometries.

The continuous contact force models gain widespread applications in multibody systems dynamics due to their computational simplicity and efficiency [25,40]. For the accurate description of the dynamic behavior of a multibody system, several aspects are crucial: the change in velocity before and after the impact, the duration time and the peak contact force. The new model shows high accuracy in these aspects. It should be noted that when the continuous contact force models are applied straightforward to flexible multibody dynamics, a wide range of speed changes may bring about pulling force when calculating the contact forces, which goes against the physical meaning of the contact force [40]. The approximate dynamic equation proposed in this paper can deal with this issue. As illustrated in Eq. (59) and Fig. 5, the contact force of the approximate dynamic equation keep positive in the whole contact process. Therefore, the new model in this paper 


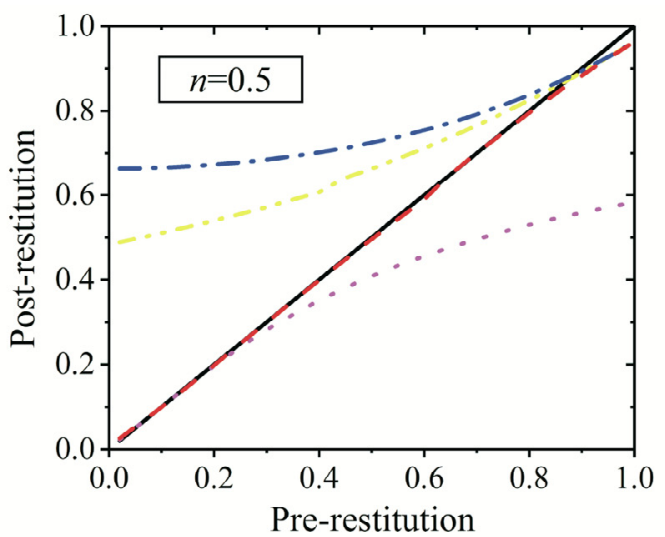

(a)

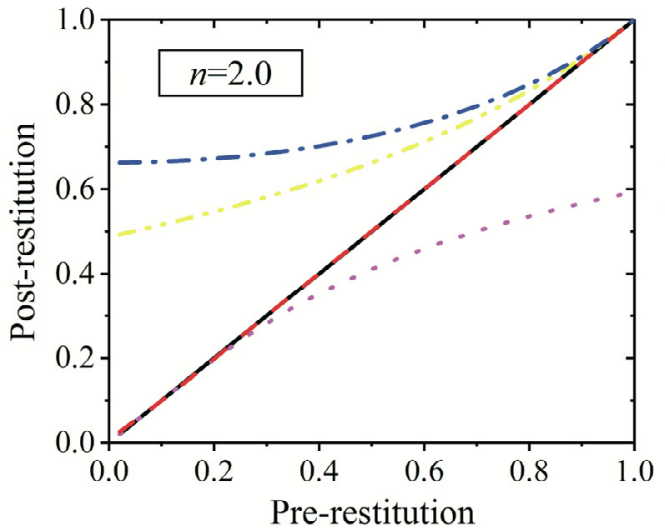

(c)

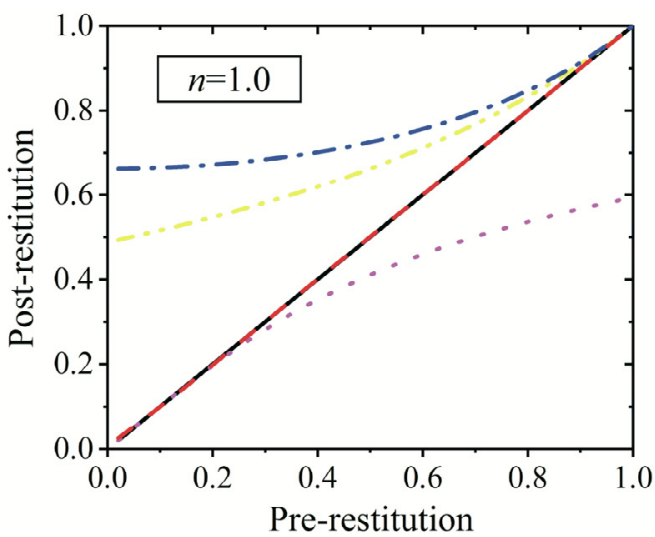

(b)

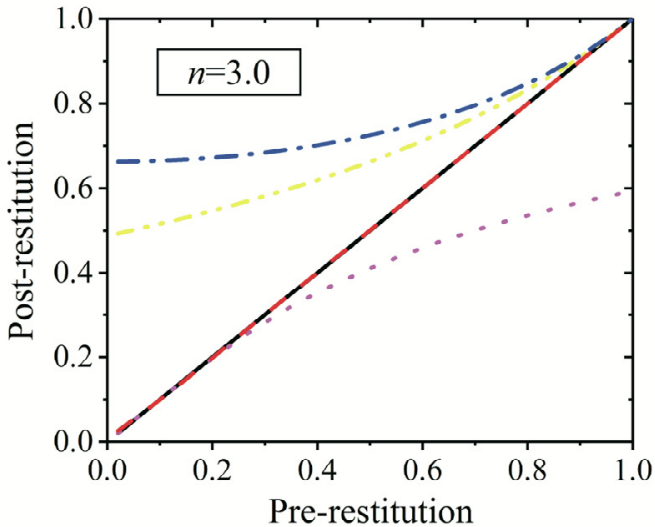

(d)

Post-restitution=Pre-restitution
_-... Hunt and Crossley model
_. . Lankarani and Nikravesh model

Fig. 9. Relation between the post- and pre-restitution coefficients for different contact models as $n=0.5,1.0,2.0$ and 3.0 .

can be expected to be applied in multibody systems to improve the numerical simulation accuracy, and on the basis of the approximate dynamic equation proposed in this paper, the continuous contact force models can be applied straightforward to flexible multibody system dynamics.

In addition to the simulation of multibody systems [18,22], the applications of the contact force models involve the modeling of granular structures [26], like, sand, clay, seeds [50], hail specimens [51], and others, i.e., the simulation of Discrete Element Method [26], structural pounding during earthquakes [52] and the contact process between barrel and bourrelet of projectile [53]. In the modern days, its application has extended to other fields [26], such as the simulation of smoothed particle hydrodynamics. There are many contact-impact phenomena with complex geometries in these fields and the model proposed in this paper may be useful for these fields, which shows high accuracy and is available for the contact problems with complex geometries.

It should be noted that the new model in this paper is proposed on the basis of the Hertz model and Hunt and Crossley model. Both models are the classic theories of contact mechanics and have provided the bases of most continuous contact force models $[1,9,10,12,21,26,27,33,34]$. However, the contact force in both models is considered as a concentrated force, as illustrated in Eqs. (1) and (2). For the long contacts within mechanical systems, the contact forces are distributed along its whole length, and multiple contact situations may arise. And the assumption of concentrated force seems to be unreasonable. In addition, according to the Hunt-Crossley model, the indentation depth is zero at the end of impact, which is not in accordance with experimental results on some viscoelastic impacts [54]. For contact detection, which is a critical aspect in contact-impact simulations, Flores et al. have been studied the contact detection algorithm for the multibody simulations with continuous contact force models and proposed an excellent methodology [55]. 


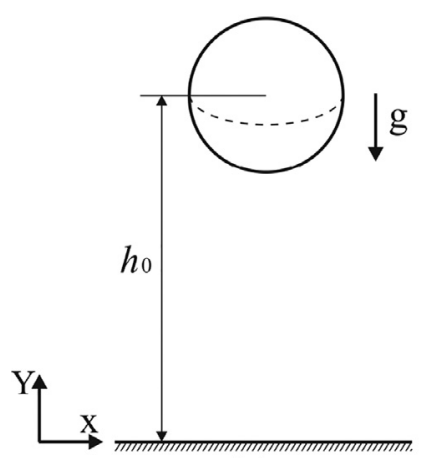

(a)

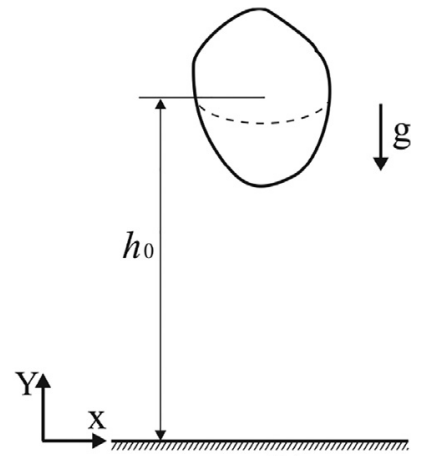

(b)

Fig. 10. Impact of falling objects: (a) an elastic sphere; (b) an elastic object with complex geometries.

Table 4

The basic parameters of the simulations.

\begin{tabular}{ll}
\hline Characteristics & Values \\
\hline Mass of the falling object & $m=1.0 \mathrm{~kg}$ \\
Initial height & $h_{0}=1.0 \mathrm{~m}$ \\
Gravity acceleration & $g=9.8 \mathrm{~m} / \mathrm{s}^{2}$ \\
Equivalent stiffness of the contact & $K=1.4 \times 10^{8} \mathrm{~N} / \mathrm{m}^{3 / 2}$ \\
Initial velocity of the falling object & $v_{0}=0 \mathrm{~m} / \mathrm{s}$ \\
\hline
\end{tabular}

Table 5

Post- and pre-restitution coefficients for contact force models.

\begin{tabular}{llllll}
\hline Pre-restitution coefficient & \multicolumn{2}{l}{$\mathrm{c}_{\mathrm{r}}=0.9000$} & & \multicolumn{2}{l}{$\mathrm{c}_{\mathrm{r}}=0.2000$} \\
\cline { 2 - 3 } \cline { 5 - 6 } Post-restitution coefficient & $\mathrm{c}_{\mathrm{r}}$ & Relative error [\%] & & $\mathrm{c}_{\mathrm{r}}$ & Relative error [\%] \\
\hline Hunt and Crossley [27] & 0.9088 & -0.98 & & 0.5459 & -172.93 \\
Lankarani and Nikravesh [21] & 0.9130 & -1.44 & & 0.6715 & -235.75 \\
Kuwabara and Kono [32] & 0.9999 & -11.10 & & 0.9992 & -399.60 \\
Flores et al. [10] & 0.8937 & 0.71 & & 0.1538 & 23.13 \\
Gharib and Hurmuzlu [33] & 0.5659 & 37.13 & & 0.1953 & 2.36 \\
Hu and Guo [12] & 0.8997 & 0.04 & & 0.1638 & 18.09 \\
Shen et al. [34] & 0.9007 & -0.08 & & 0.1943 & 2.84 \\
The model of this paper & 0.8998 & 0.03 & 0.1985 & 0.74 \\
\hline
\end{tabular}

\section{Numerical examples}

To conduct a more detailed analysis of the contact model proposed in this study and evaluate the influences of the contacting surface geometries on the system dynamic responses, the contact-impact of a free-falling object is simulated. As shown in Fig. 10, an elastic body made of PTFE is released from the initial position under the influence of gravity only and collides with the ground, which is assumed to be rigid and stationary. The basic parameters of the simulation are given in Table $4[10,12]$. The restitution coefficients are set as 0.9 and 0.2 to compare the dynamic responses of the system with high and low restitution coefficients.

First, an elastic sphere with a radius of $0.1 \mathrm{~m}$ is considered, and the exponent $n$ is set 1.5 , as illustrated in Fig. 10a. All contact models listed in Table 1 are utilized for modeling the first contact-impact event of the free-falling sphere. The time histories of the deformation and contact force of the ball during the contact-impact process, and the relationship between contact force and deformation [56] are demonstrated in Figs. 11-16. In addition, Table 5 provides the values of post- and pre-restitution coefficients of the eight different contact force models.

Figs. 11-13 and Table 5 show that when the restitution coefficient is 0.9 , the simulation results on the foundation of the models are basically the same, except for the Kuwabara and Kono model [32] and Gharib and Hurmuzlu model [33]. In Fig. 13, we observe that Kuwabara and Kono model dissipate little energy as the expressions of Kuwabara and Kono model presented in Table 1 are developed for $c_{r} \approx 1$. It can also be seen that Gharib and Hurmuzlu model dissipate more energy and exhibit larger hysteresis loops than other models as $c_{r}=0.9$, because this model is proposed for a low coefficient of restitution. As shown in Table 5, the differences between the post-restitution coefficients calculated by using each model and the pre-restitution coefficients are consistent with the previous analysis in Section 5.

As illustrated in Figs. 14-16 and Table 5, when the restitution coefficient is 0.2, the simulation results calculated by the Flores et al. model, Gharib and Hurmuzlu model, Hu and Guo model, Shen et al. model and the model described in this 


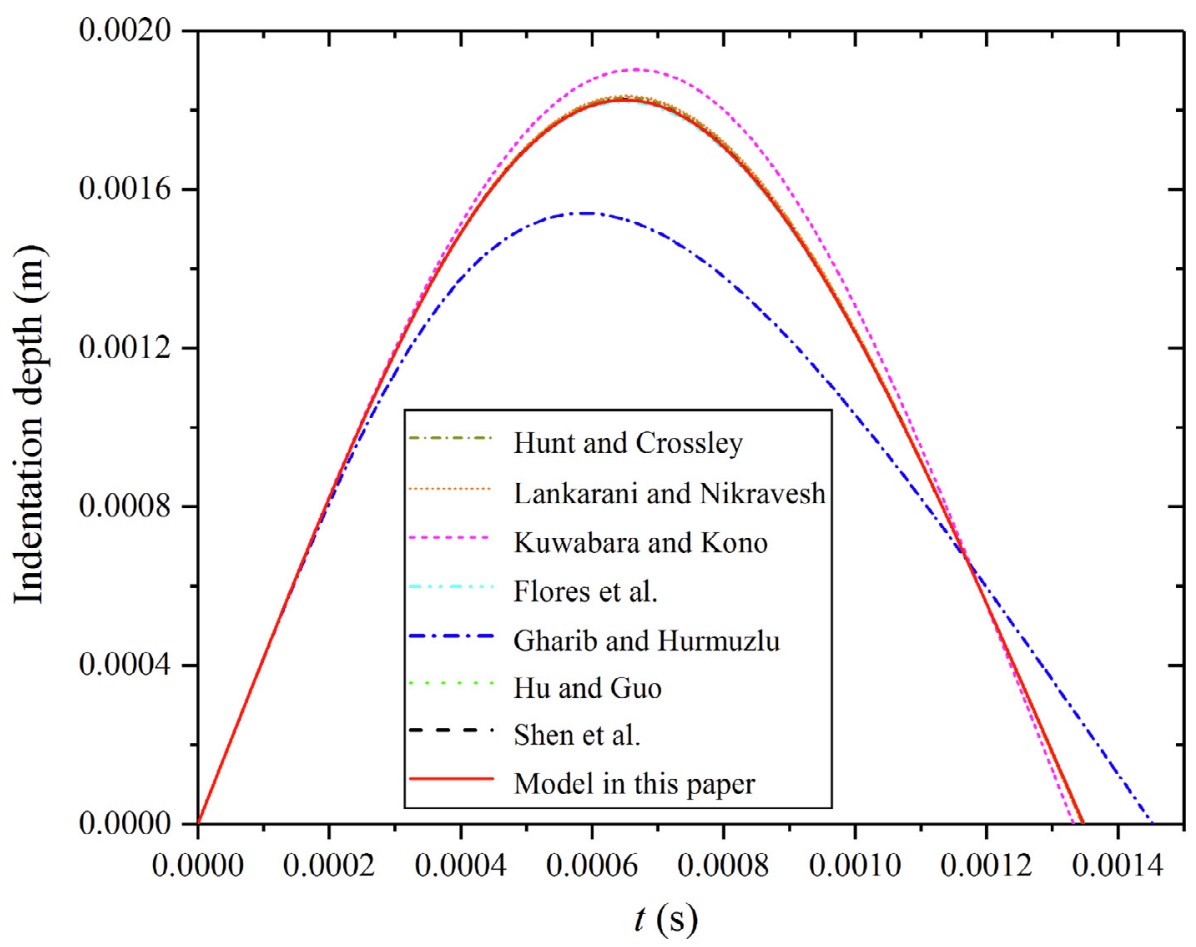

Fig. 11. Time history of the indentation depth for different contact force models with $c_{r}=0.9$.

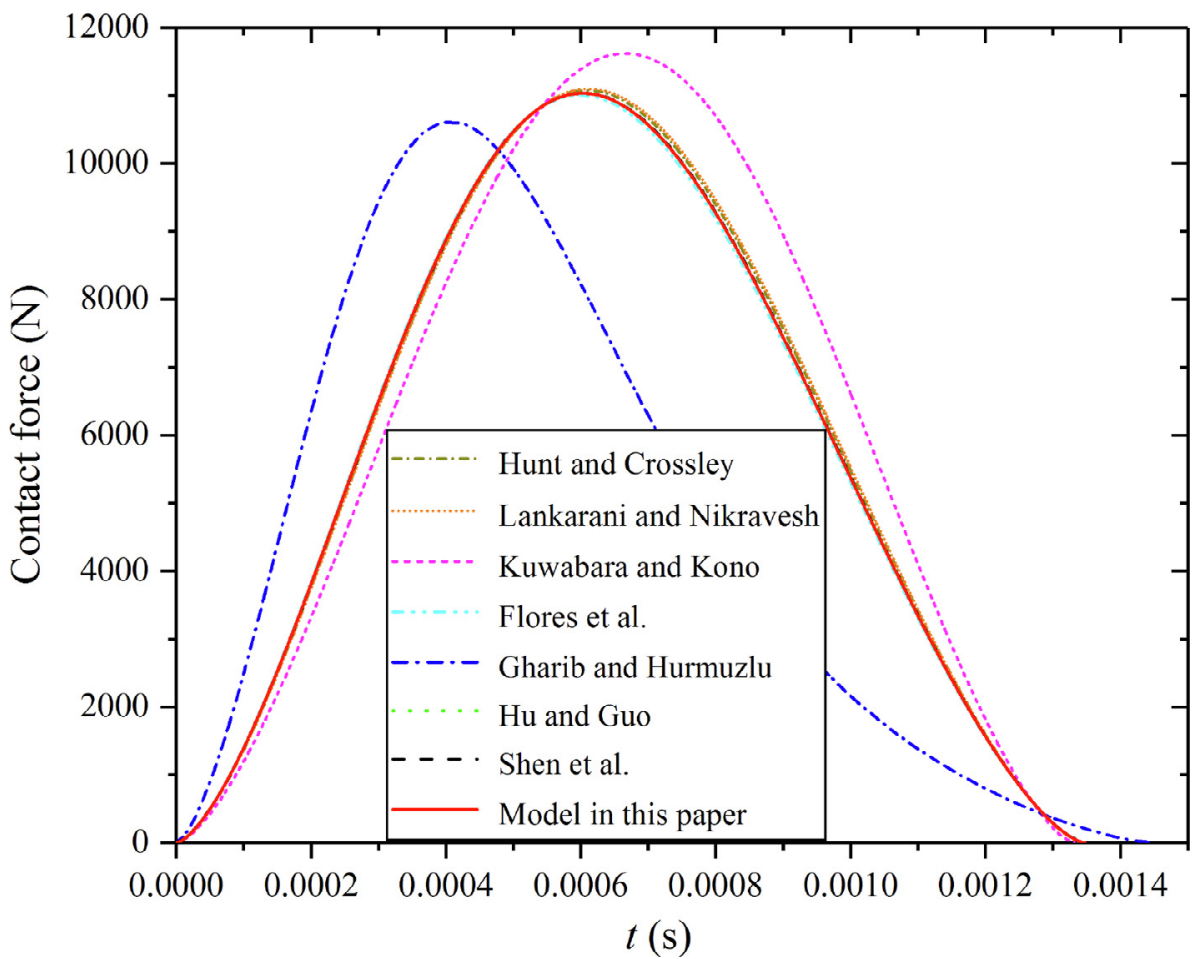

Fig. 12. Time history of the contact force for different contact force models with $c_{r}=0.9$. 


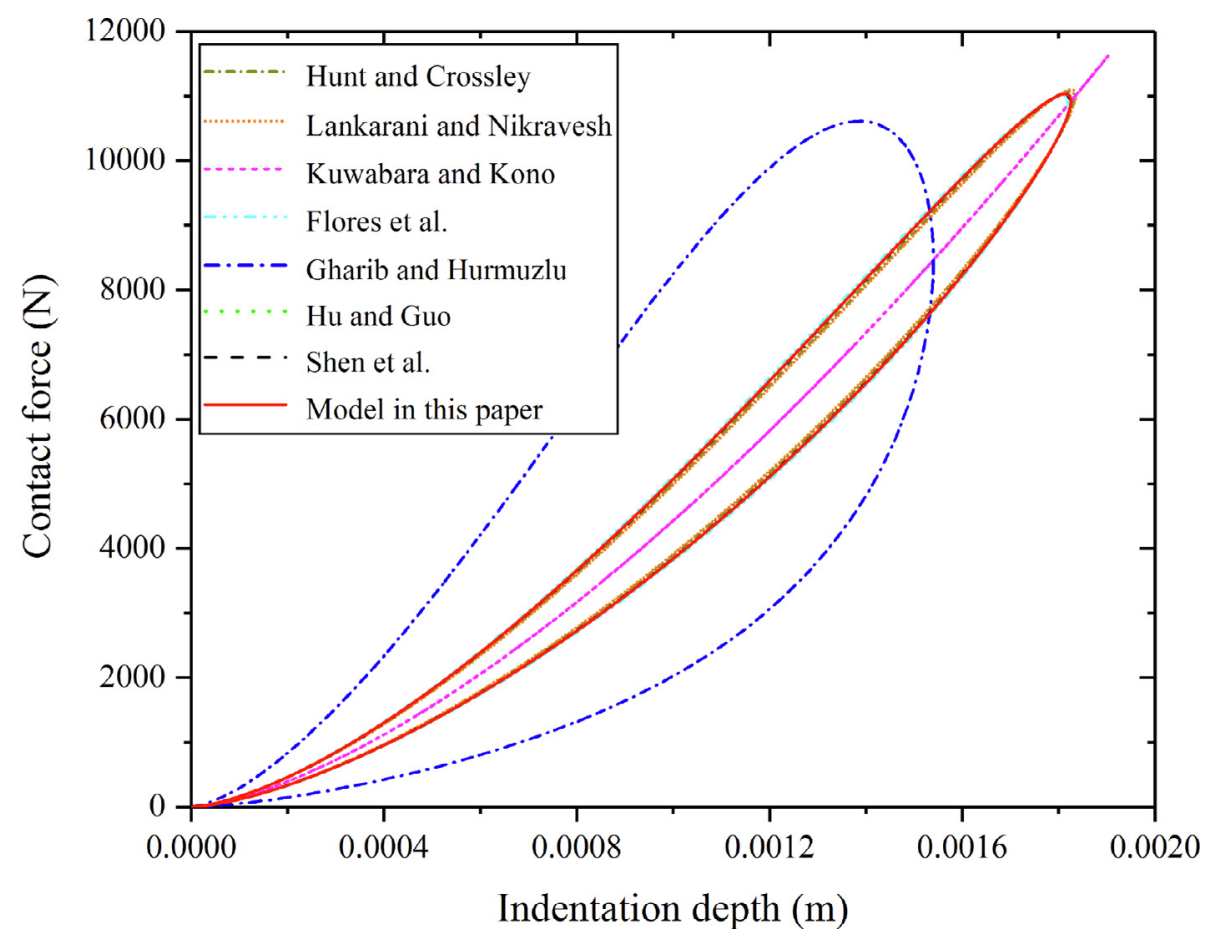

Fig. 13. Relationship between Contact force and indentation depth for different contact force models with $c_{r}=0.9$.

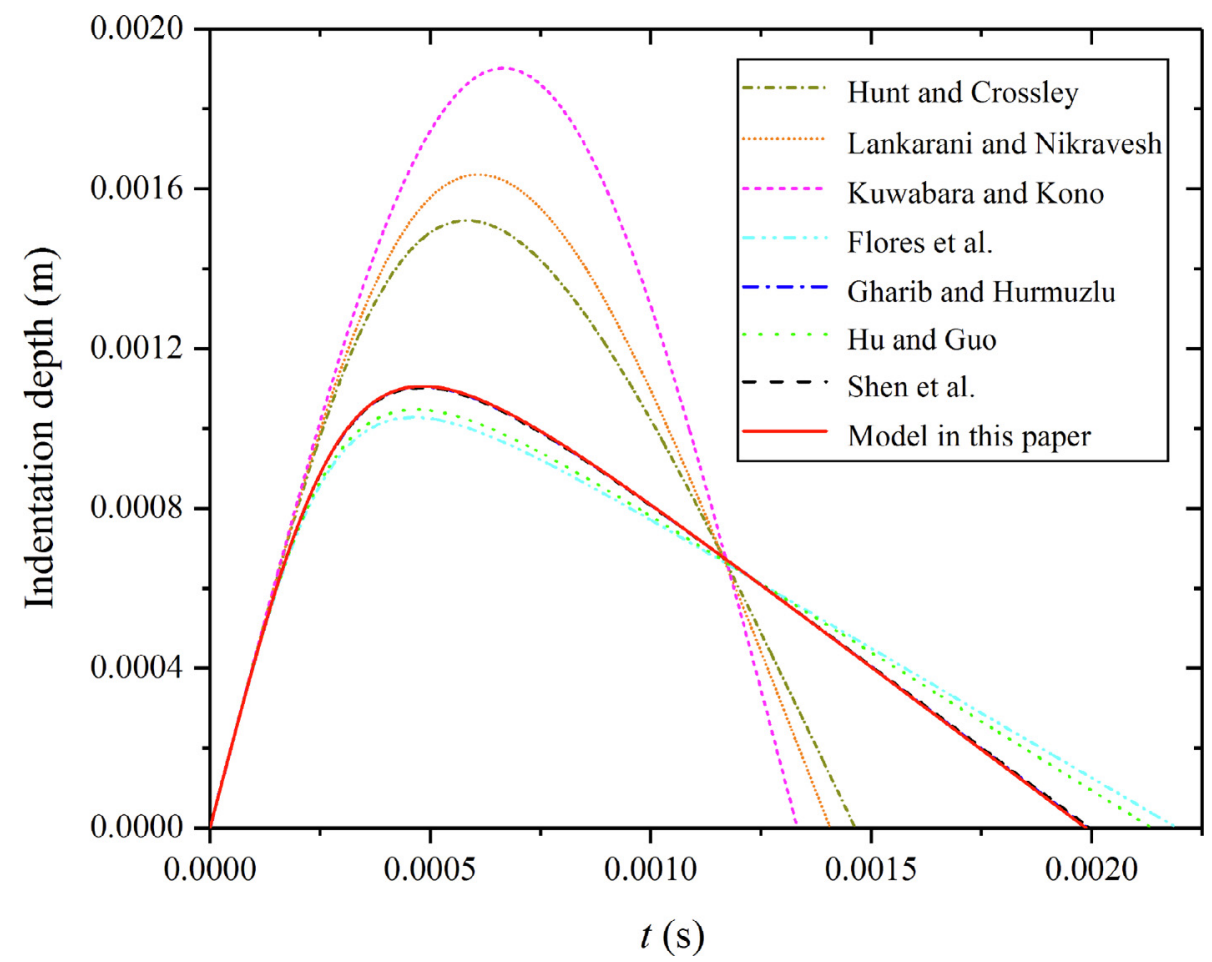

Fig. 14. Time history of the indentation depth for different contact force models with $c_{r}=0.2$. 


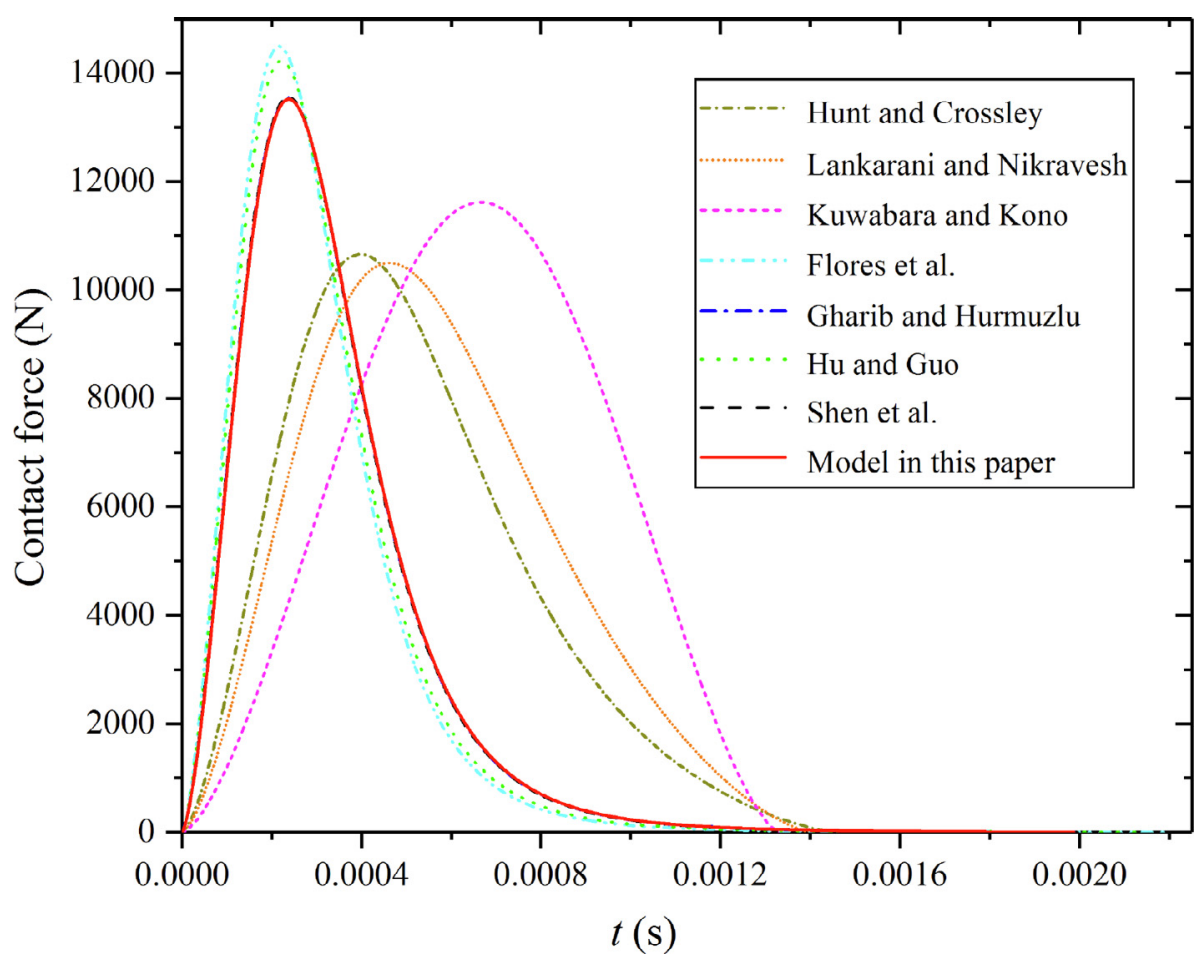

Fig. 15. Time history of the contact force for different contact force models with $c_{r}=0.2$.

paper tend to be consistent, with only small differences. In contrast, calculations of the Lankarani and Nikravesh model and Hunt and Crossley model and Kuwabara and Kono model lead to greater indentation depths, smaller contact forces, and shorter contact durations. As demonstrated in Table 5, it can be seen that the post-restitution coefficients calculated based on Flores et al. model, Gharib and Hurmuzlu model, Hu and Guo model, Shen et al. model and the model described in this paper are relatively close to the pre-restitution coefficients, while the post-restitution coefficients obtained by Lankarani and Nikravesh model, Hunt and Crossley model and Kuwabara and Kono model are quite different from the pre-restitution coefficients. It can be inferred that the Gharib and Hurmuzlu model is suitable for contact problems with a low coefficient of restitution and the Flores et al. model, Hu and Guo model, Shen et al. model and the model described in this paper are suitable for the case of not only low energy dissipation during the contact process but also higher energy dissipation, in other words, these four models exhibit good performance within the entire range of the coefficient of restitution.

Then, the model described in this paper is applied to simulate the contact-impact of a free-falling object with more complex contacting surface, as illustrated in Fig. 10b. The numerical simulations are conducted with the initial condition as shown in Table 4, and the exponent $n$, which describes the topological properties of the contacting surfaces, is set to 1.0, $1.2,1.5$ and 1.8 .

As illustrated in Figs. 17-22, under the same other conditions, the simulation results of the collision process are obviously different under small differences in $n$. With the increase in $n$, the indentation depth and contact-impact time increase, and the maximum contact force decreases. Moreover, it can be seen that with the reduction in the restitution coefficient, the amount of energy loss increases, and the symmetry between the compression phase and the restitution phase reduces. Figs. 19 and 22 show that the differences in the amount of energy loss under different values of exponent $n$ are small, which means that the influence of the topological properties of contacting surfaces on the amount of energy loss is not significant. As shown in Figs. 17 and 18, all the curves show approximate symmetry. Figs. 20 and 21 show that when $c_{r}=0.2$, the duration of the restitution phase is significantly longer than that of the compression phase, and the differences between the occurrence time of the maximum contact force and the maximum indentation depth during the contact-impact process are more obvious. During the restitution phase, deformation gradually recovers, and the contact force decreases. As illustrated in Figs 20 and 21, when $c_{r}=0.2$, the contact force is almost zero in the latter stage of the restitution phase, while the deformation is still obvious. This phenomenon is consistent with the experimental phenomenon to some extent [57]. The above analysis also supports the significance of developing the contact force model for contact problems with complex geometries. 


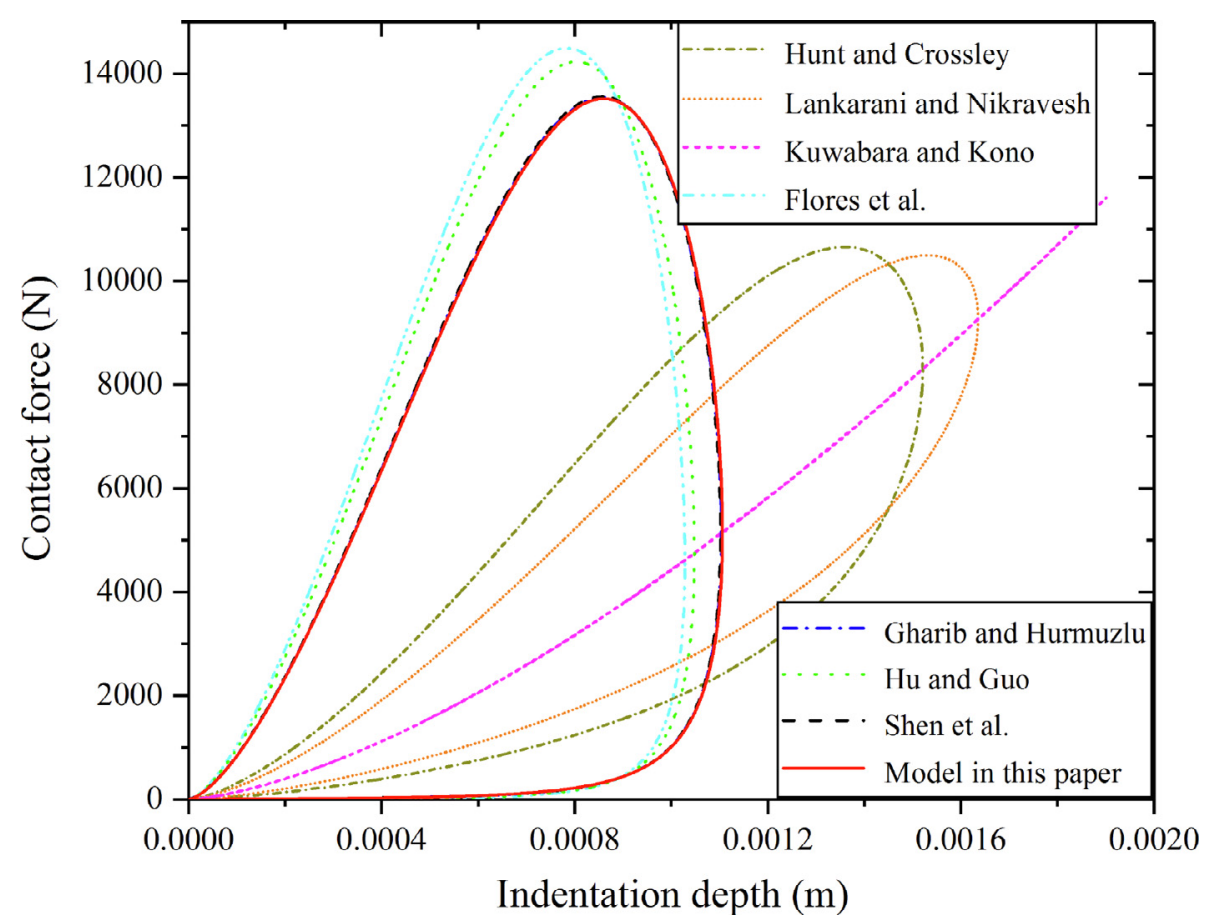

Fig. 16. Relationship between Contact force and indentation depth for different contact force models with $c_{r}=0.2$.

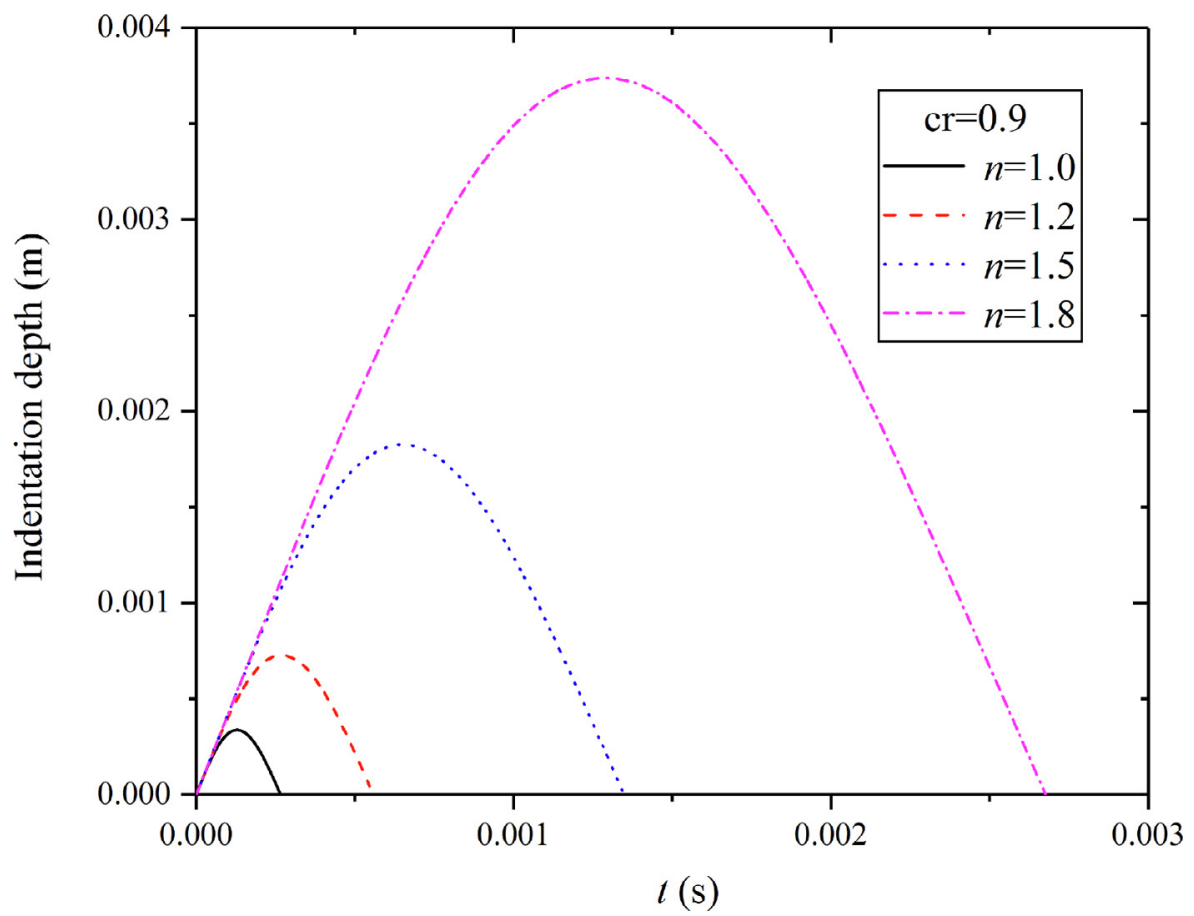

Fig. 17. Time history of the indentation depth for different contacting surfaces with $c_{r}=0.9$. 


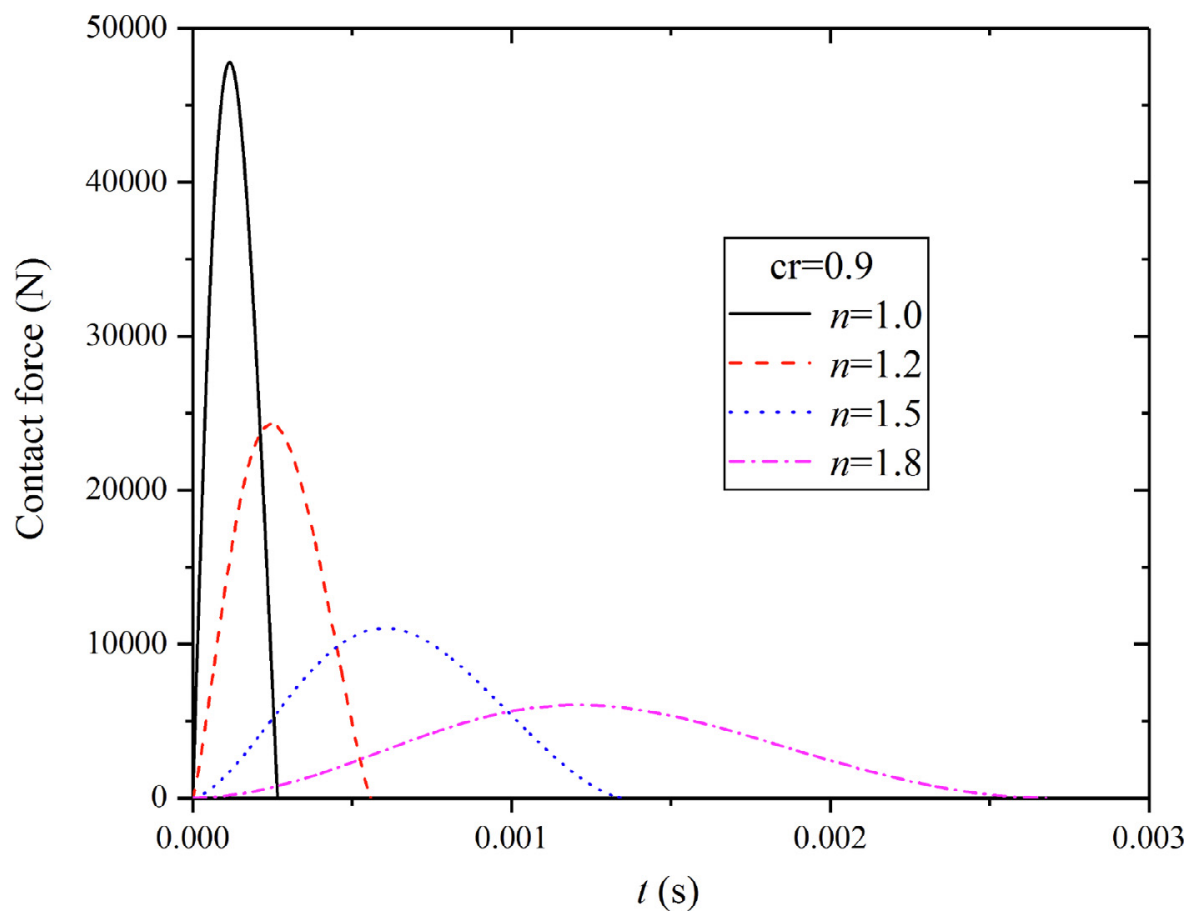

Fig. 18. Time history of the contact force for different contacting surfaces with $c_{r}=0.9$.

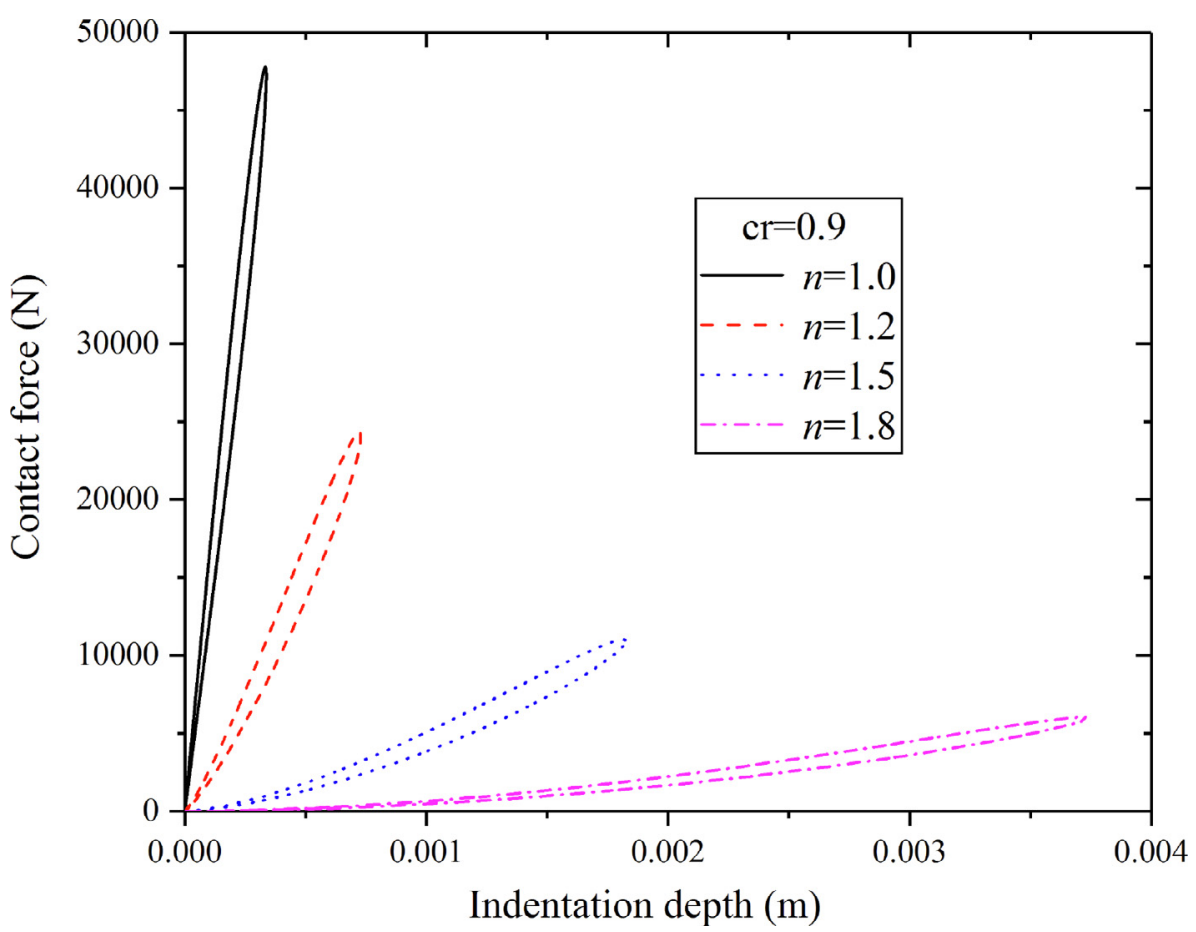

Fig. 19. Relationship between Contact force and indentation depth for different contacting surfaces with $c_{r}=0.9$. 


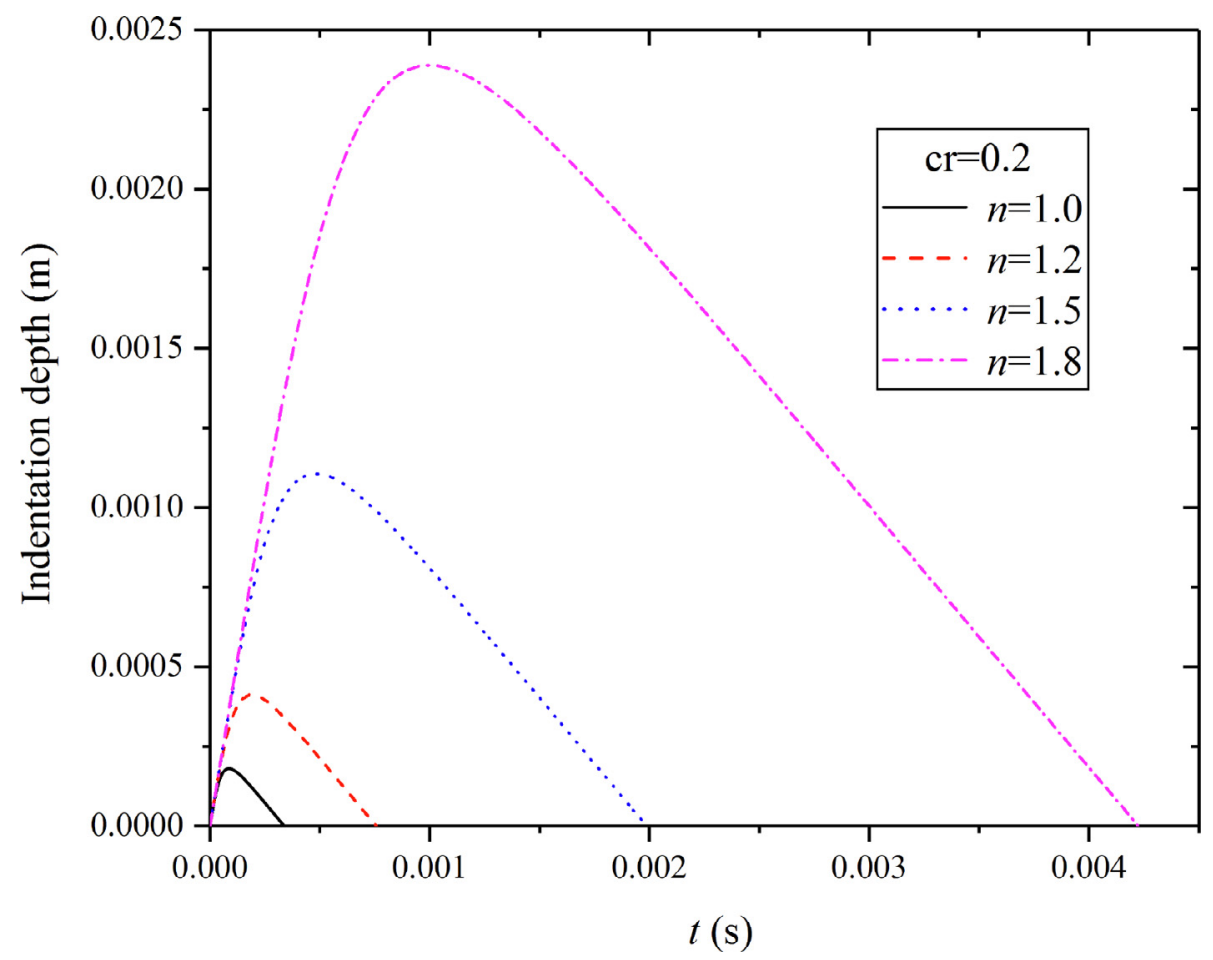

Fig. 20. Time history of the indentation depth for different contacting surfaces with $c_{r}=0.2$.

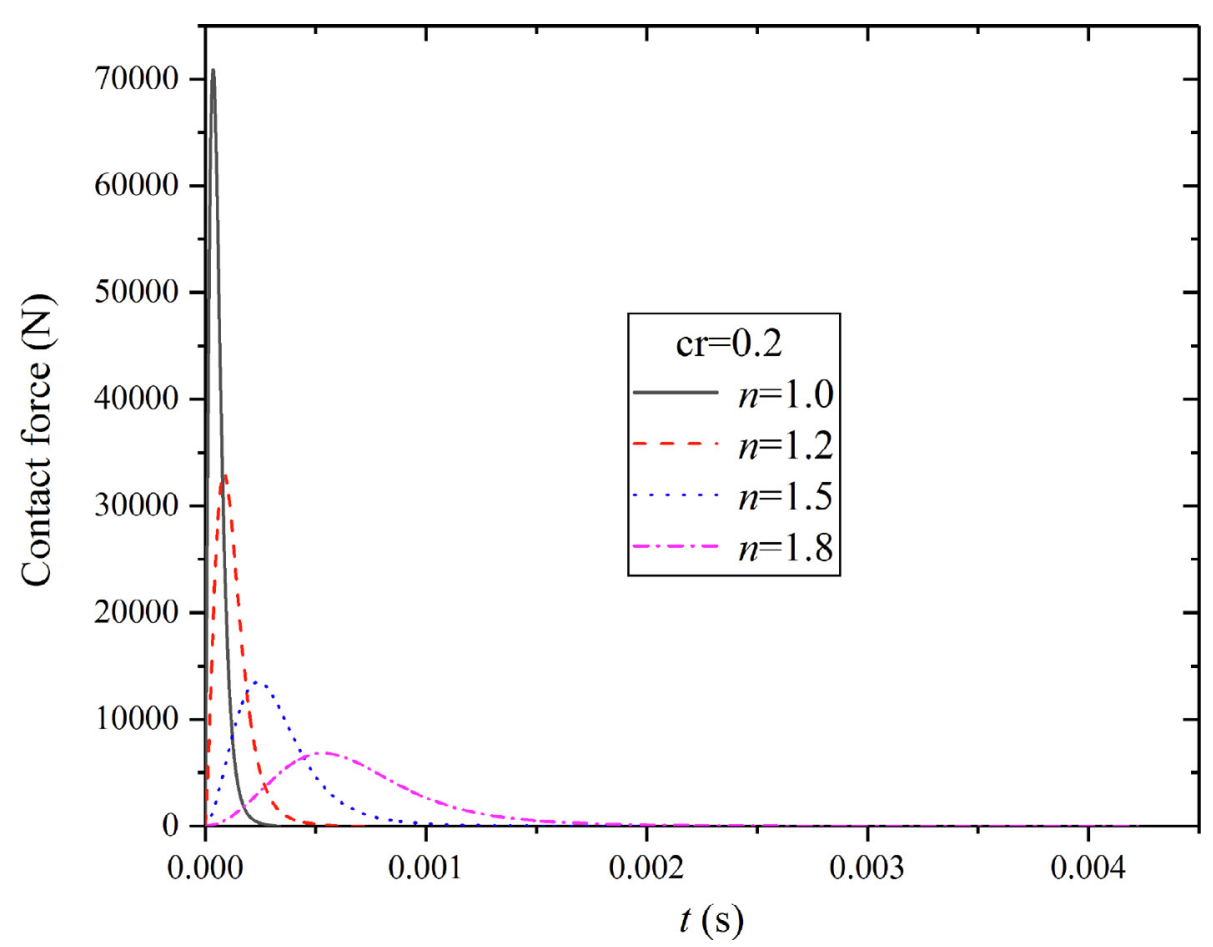

Fig. 21. Time history of the contact force for different contacting surfaces with $c_{r}=0.2$. 


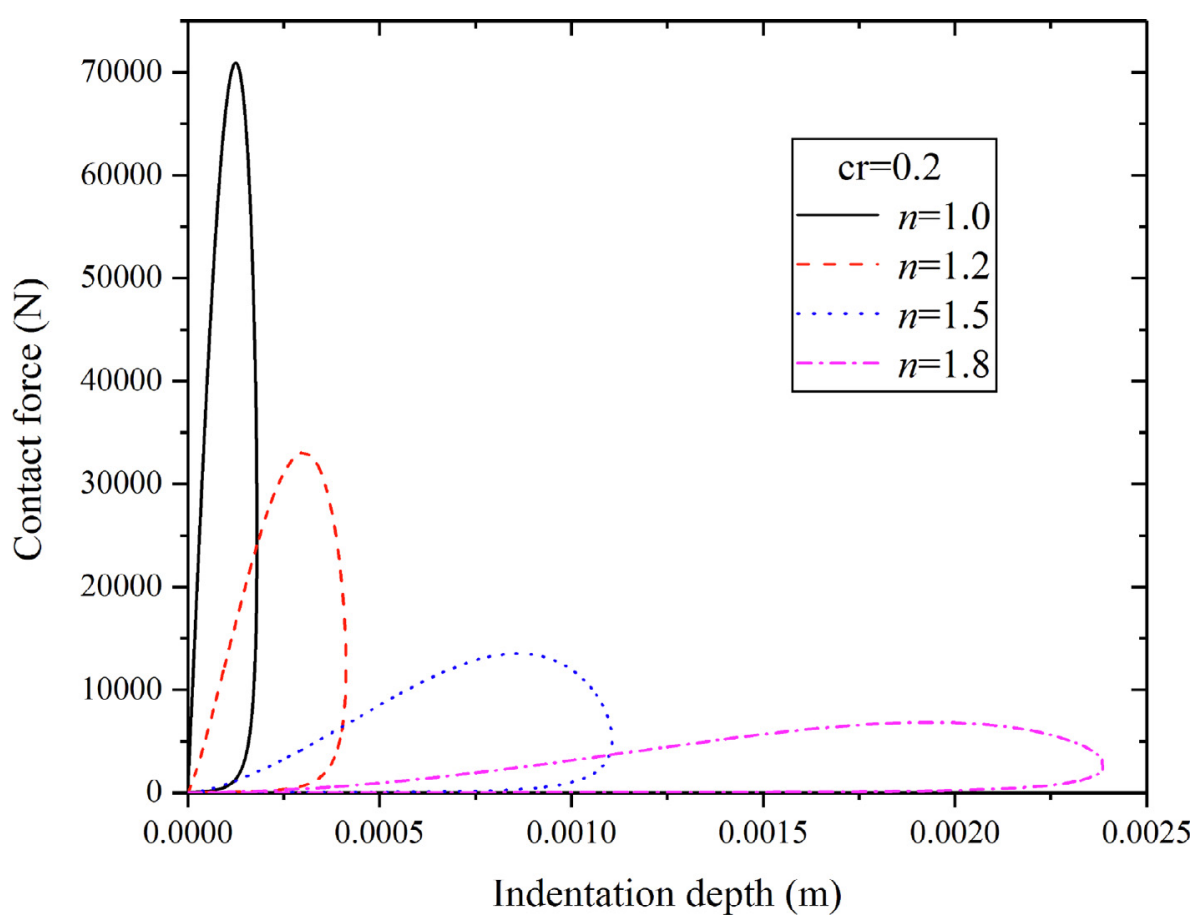

Fig. 22. Relationship between Contact force and indentation depth for different contacting surfaces with $c_{r}=0.2$.

\section{Conclusions}

A new continuous contact force model is proposed for impact analysis in multibody dynamics with complex geometries and with energy dissipation. The main difficulty of this research is that it is almost impossible to obtain an analytical solution from the system dynamic equation. An approximate dynamic equation is developed by introducing equivalent velocity. The analysis and simulations illustrate that the approximate dynamic equation with properly chosen equivalent velocities can accurately approximate the system dynamic equation. An approximate function between the deformation velocity and indentation depth is obtained considering the approximate dynamic equation and is utilized to calculate the energy loss due to the damping force. Then, the expression of the hysteresis damping parameter is derived by combining energy balance and the law of conservation of linear momentum. Considering the deviation between the approximate equation and the system dynamic equation, the primary formula of the hysteresis damping parameter is modified by a nondimensional method, and a more accurate model is developed.

The capability of the new model is validated and compared by published experimental data and simulation results of eight different contact models. The new model shows high accuracy on the consistency of post- and pre-restitution coefficients, contact duration time and peak contact force. The numerical results also reveal that the influences of the geometry of the contacting surfaces on the dynamic system responses are significant, which demonstrates that the presentation of the new model is of great value. The new model provides a valuable method with which to simulate contacting problems with complex contacting surface geometries and may be useful for multibody system simulation and other fields.

\section{Declaration of Competing Interest}

This is a pure academic research paper. There is not any conflict of interests to any person/ organizations/groups.

\section{Acknowledgements}

This research was supported by the National Key Research and Development Plan of China [grant number 2019YFB1309600]; the National Natural Science Foundation of China [grant numbers 11702294 and 51775002]; the Joint Program of Beijing Municipal Foundation and Education Commission [grant number KZ202010009015]; and the Beijing Natural Science Foundation [grant number 3194047]. The author would like to thank the anonymous reviewers for their insightful comments and suggestions on an earlier draft of this paper, and would also like to thank to American Journal Experts for their English corrections. 


\section{References}

[1] S. Luka, S. Janko, B. Miha, A review of continuous contact-force models in multibody dynamics, Int. J. Mech. Sci. 145 (2018) 171-187, doi:10.1016/j. ijmecsci.2018.07.010.

[2] P. Flores, J. Ambrósio, J.C. Claro, H.M. Lankarani, Translational joints with clearance in rigid multibody systems, J. Comput. Nonlinear Dyn. 3 (1) (2008) 011007, doi:10.1115/1.2802113.

[3] Q. Tian, P. Flores, H.M. Lankarani, A comprehensive survey of the analytical, numerical and experimental methodologies for dynamics of multibody mechanical systems with clearance or imperfect joints, Mech. Mach. Theory 122 (2018) 1-57, doi:10.1016/j.mechmachtheory.2017.12.002.

[4] Q. Wang, Q. Tian, H. Hu, Dynamic simulation of frictional multi-zone contacts of thin beams, Nonlinear Dynam 83 (4) (2015) 1-19, doi:10.1007/ s11071-015-2456-8.

[5] Q. Tian, Y. Zhang, L. Chen, J. Yang, Simulation of planar flexible multibody systems with clearance and lubricated revolute joints, Nonlinear Dyn. 60 (4) (2010) 489-511, doi:10.1007/s11071-009-9610-0.

[6] J. Zhang, Q. Wang, Modeling and simulation of a frictional translational joint with a flexible slider and clearance, Multibody Syst. Dyn. 38 (4) (2016) 367-389, doi:10.1007/s110-015-9474-7.

[7] X. Zheng, J. Li, Q. Wang, et al., A methodology for modeling and simulating frictional translational clearance joint in multibody systems including a flexible slider part, Mech. Mach. Theory 142 (2019) 103603, doi:10.1016/j.mechmachtheory.2019.103603.

[8] C.S. Koshy, P. Flores, H.M. Lankarani, Study of the effect of contact force model on the dynamic response of mechanical systems with dry clearance joints: computational and experimental approaches, Nonlinear Dynam. 73 (1-2) (2013) 325-338, doi:10.1007/s11071-013-0787-x.

[9] J. Alves, N. Peixinho, M.T. Da Silva, et al., A comparative study of the viscoelastic constitutive models for frictionless contact interfaces in solids, Mech. Mach. Theory 85 (2015) 172-188, doi:10.1016/j.mechmachtheory.2014.11.020.

[10] P. Flores, M. Machado, M.T. Silva, et al., On the continuous contact force models for soft materials in multibody dynamics, Multibody Syst. Dyn. 25 (3) (2011) 357-375, doi:10.1007/s11044-010-9237-4.

[11] G. Gilardi, I. Sharf, Literature survey of contact dynamics modelling, Mech. Mach. Theory 37 (10) (2002) 1213-1239, doi:10.1016/s0094-114x(02) 00045-9.

[12] S. Hu, X. Guo, A dissipative contact force model for impact analysis in multibody dynamics, Multibody Syst. Dyn. 35 (2) (2015) 131-151, doi:10.1007/ s11044-015-9453-z.

[13] R. Seifried, W. Schiehlen, P. Eberhard, The role of the coefficient of restitution on impact problems in multi-body dynamics, Proc. Inst. Mech. Eng., Proc., Part K, J. Multi-Body Dyn. 224 (3) (2010) 279-306, doi:10.1243/14644193jmbd239.

[14] A. Kaveh, V.R. Mahdavi, Colliding bodies optimization: a novel meta-heuristic method, Comput. Struct. 139 (2014) 18-27, doi:10.1016/j.compstruc.2014. 04.005.

[15] K. Kardel, H. Ghaednia, A.L. Carrano, et al., Experimental and theoretical modeling of behavior of 3d-printed polymers under collision with a rigid rod, Addit. Manuf. 14 (2017) 87-94, doi:10.1016/j.addma.2017.01.004.

[16] Y. Lin, R.T. Haftka, N.V. Queipo, B.J. Fregly, Surrogate articular contact models for computationally efficient multibody dynamic simulations, Med. Eng. Phys. 32 (6) (2010) 584-594, doi:10.1016/j.medengphy.2010.02.008.

[17] M. Anitescu, F.A. Potra, D.E. Stewart, Time-stepping for three-dimensional rigid body dynamics, Comput. Methods Appl. Math. 177 (3-4) (1999) 183197, doi:10.1016/s0045-7825 (98)00380-6.

[18] P. Flores, R. Leine, C. Glocker, Modeling and analysis of planar rigid multibody systems with translational clearance joints based on the non-smooth dynamics approach, Multibody Syst. Dyn. 23 (2) (2010) 165-190, doi:10.1007/978-90-481-9971-6_6.

[19] J. Pang, D. Stewart, Differential variational inequalities, Math. Program. 113 (2) (2008) 345-424, doi:10.1007/s10107-006-0052-x.

[20] A. Tasora, D. Negrut, M. Anitescu, Large-scale parallel multi-body dynamics with frictional contact on the graphical processing unit, Proc. Inst. Mech. Eng., Proc., Part K, J. Multi-Body Dyn. 222 (4) (2008) 315-326, doi:10.1243/14644193jmbd154.

[21] H.M. Lankarani, P.E. Nikravesh, A contact force model with hysteresis damping for impact analysis of multibody systems, J. Mech. Des. 112 (3) (1990) 369-376, doi:10.1115/1.2912617.

[22] X. Zheng, F. Zhang, Q. Wang, Modeling and simulation of planar multibody systems with revolute clearance joints considering stiction based on an LCP method, Mech. Mach. Theory 130 (2018) 184-202, doi:10.1016/j.mechmachtheory.2018.08.017.

[23] R. Zhang, Y. Yu, Q. Wang, et al., An improved implicit method for mechanical systems with set-valued friction, Multibody Syst. Dyn. (2019) 1-28, doi:10.1007/s11044-019-09713-0.

[24] K.L. Johnson, One hundred years of Hertz contact, Proc. Inst. Mech. Eng. 196 (1982) 363-378, doi:10.1243/pime_proc_1982_196_039_02.

[25] M. Machado, P. Moreira, P. Flores, H.M. Lankarani, Compliant contact force models in multibody dynamics: evolution of the Hertz contact theory, Mech. Mach. Theory 53 (2012) 99-121, doi:10.1016/j.mechmachtheory.2012.02.010.

[26] A. Banerjee, A. Chanda, R. Das, Historical origin and recent development on normal directional impact models for rigid body contact simulation: a critical review, Arch. Comput. Method E 24 (2) (2017) 397-422, doi:10.1007/s11831-016-9164-5.

[27] K.H. Hunt, F.R.E. Crossley, Coefficient of restitution interpreted as damping in vibroimpact, J. Appl. Mech. 42 (2) (1975) 440-445, doi:10.1115/1.3423596.

[28] R.G. Herbert, D.C. Mcwhannell, Shape and frequency composition of pulses from an impact pair, J. Eng. Ind. 99 (3) (1977) 513-518, doi:10.1115/1. 3439270.

[29] T.W. Lee, A.C. Wang, On the dynamics of intermittent-motion mechanisms. Part 1: dynamic model and response, J. Mech. Transm.-T. Asme. 105 (3) (1983) 534-540, doi:10.1115/1.3267392.

[30] Y. Gonthier, J. Mcphee, C. Lange, J. Piedbœuf, A regularized contact model with a symmetric damping and dwell-time dependent friction, Multibody Syst. Dyn. 11 (3) (2004) 209-233, doi:10.1023/b:mubo.0000029392.21648.bc.

[31] Z.Y. Qin, Q.S. Lu, Analysis of impact process model based on restitution coefficient, J, Dyn. Control 4 (2006) 294-298, doi:10.3969/j.issn.1672-6553. 2006.04.002.

[32] G. Kuwabara, K. Kono, Restitution coefficient in a collision between two spheres, Jpn. J. Appl. Phys. 26 (8) (1987) 1230-1233, doi:10.1143/JJAP.26.1230.

[33] M. Gharib, Y. Hurmuzlu, A new contact force model for low coefficient of restitution impact, J. Appl. Mech.-T. Asme. 79 (6) (2012), doi:10.1115/1. 4006494.

[34] Y. Shen, D. Xiang, X. Wang, et al., A contact force model considering constant external forces for impact analysis in multibody dynamics, Multibody Syst. Dyn. 44 (4) (2018) 397-419, doi:10.1007/s11044-018-09638-0.

[35] H. Safaeifar, A. Farshidianfar, A new model of the contact force for the collision between two solid bodies, Multibody Syst. Dyn. 6 (2020), doi:10.1007/ s11044-020-09732-2.

[36] J. Yu, J.K. Chu, Y. Li, L. Guan, An improved compliant contact force model using a piecewise function for impact analysis in multibody dynamics. Proc. Inst. Mech. Eng., Proc., Part K, J. Multi-Body Dyn. 10.1177/1464419319900874.

[37] M. Poursina, P.E. Nikravesh, Characterization of the damping coefficient in the continuous contact model, ASME International Design Engineering Technical Conferences and Computers and Information in Engineering Conference, 2019, doi:10.1115/DETC2019-97476.

[38] A.S. Carvalho, J.M. Martins, Exact restitution and generalizations for the Hunt-Crossley contact model, Mech. Mach. Theory 139 (2019) 174-194, doi:10. 1016/j.mechmachtheory.2019.03.028.

[39] B. Jian, G.M. Hu, Z.Q. Fang, et al., Comparative behavior of damping terms of viscoelastic contact force models with consideration on relaxation time, Powder Technol 356 (2019) 735-749, doi:10.1016/j.powtec.2019.08.110.

[40] X.G. Zhang, Z.H. Qi, G. Wang, S.D. Guo, Model smoothing method of contact-impact dynamics in flexible multibody systems, Mech. Mach. Theory 138 (2019) 124-148, doi:10.1016/j.mechmachtheory.2019.03.039.

[41] K.L. Johnson, Contact Mechanics, 1st Ed., Cambridge university press, London, 1985. 
[42] V.L. Popov, Contact Mechanics and Friction, 1st Ed., Springer, Berlin Heidelberg, Berlin, 2010

[43] S. Ken, Newton's cradle versus nonbinary collisions, Phys. Rev. Lett. 104 (12) (2010) 124302, doi:10.1103/physrevlett.104.124302.

[44] H. Jongbae, Universal power-law decay of the impulse energy in granular protectors, Phys. Rev. Lett. 94 (10) (2005) 108001, doi:10.1103/physrevlett.94. 108001.

[45] F. Zhang, Y. Yu, Q. Wang, et al., A terrain-adaptive robot prototype designed for bumpy-surface exploration, Mech. Mach. Theory 141 (2019) 213-225, doi:10.1016/j.mechmachtheory.2019.07.008.

[46] A. He, J.S. Wettlaufer, Hertz beyond belief, Soft Matter 10 (13) (2014) 2264-2269, doi:10.1039/c3sm53063a.

[47] S. Shivaswamy, Modeling contact forces and energy dissipation during impact in multibody mechanical systems, Wichita State University, Wichita, Kansas, 1997.

[48] H.M. Lankarani, P.E. Nikravesh, Continuous contact force models for impact analysis in multibody systems, Nonlinear Dyn 5 (1994) 193-207, doi:10. $1007 /$ BF00045676.

[49] Y. Zhang, I. Sharf, Validation of nonlinear viscoelastic contact force models for low speed impact, J. Appl. Mech.-T. Asme. 76 (5) (2009), doi:10.1115/1. 3112739.

[50] J. Horabik, M. Beczek, R. Mazur, et al., Determination of the restitution coefficient of seeds and coefficients of visco-elastic Hertz contact models for DEM simulations, Biosyst. Eng. 161 (2017) 106-119, doi:10.1016/j.biosystemseng.2017.06.009.

[51] J. Sun, N. Lam, L. Zhang, et al., A note on Hunt and Crossley model with generalized visco-elastic damping, Int. J. Impact Eng. 121 (2018) 151-156, doi:10.1016/j.ijimpeng.2018.07.007.

[52] R. Jankowski, Non-linear viscoelastic modelling of earthquake-induced structural pounding, Earthq. Eng. Struct. D. 34 (6) (2005) 595-611, doi:10.1002/ eqe.434.

[53] J. Ma, G. Chen, L. Ji, et al., A general methodology to establish the contact force model for complex contacting surfaces, Mech. Syst. Signal PR. 140 (2020) 106678, doi:10.1016/j.ymssp.2020.106678.

[54] M.V. Zeebroeck, E. Tijskens, P.V. Liedekerke, et al., Determination of the dynamical behaviour of biological materials during impact using a pendulum device, J. Sound Vib. 266 (3) (2003) 465-480, doi:10.1016/s0022-460x(03)00579-0.

[55] P. Flores, J. Ambrósio, On the contact detection for contact-impact analysis in multibody systems, Multibody Syst. Dyn. 24 (2010) 255-280, doi:10. 1007/s11044-010-9209-8.

[56] M. Ahmadizadeh, A.M. Shafei, M. Fooladi, A recursive algorithm for dynamics of multiple frictionless impact-contacts in open-loop robotic mechanisms, Mech. Mach. Theory 146 (2020) 1-20, doi:10.1016/j.mechmachtheory.2019.103745.

[57] S. Shivaswamy, H.M. Lankarani, Impact analysis of plates using quasi-static approach, J. Mech. Des. 119 (3) (1997) 376-381, doi:10.1115/1.2826358. 